\title{
Optimal Time-Frequency Deconvolution Filter Design for Nonstationary Signal Transmission through a Fading Channel: AF Filter Bank Approach
}

\author{
Bor-Sen Chen, Senior Member, IEEE, Yue-Chiech Chung, and Der-Feng Huang
}

\begin{abstract}
The purpose of this paper is to develop a new approach-time-frequency deconvolution filter-to optimally reconstruct the nonstationary (or time-varying) signals that are transmitted through a multipath fading and noisy channel. A deconvolution filter based on ambiguity function (AF) filter bank is proposed to solve this problem via a three-stage filter bank. First, the signal is transformed via an AF analysis filter bank so that the nonstationary (or time-varying) component is removed from each subband of the signal. Then, a Wiener filter bank is developed to remove the effect of channel fading and noise to obtain the optimal estimation of the ambiguity function of the transmitted signal in the time-frequency domain. Finally, the estimated ambiguity function of the transmitted signal in each subband is sent through an AF synthesis filter bank to reconstruct the transmitted signal. In this study, the channel noise may be time-varying or nonstationary. Therefore, the optimal separation problem of multicomponent nonstationary signals is also solved by neglecting the transmission channel.
\end{abstract}

Index Terms - Ambiguity function (AF) filter bank, fading channel, time-frequency deconvolution filter, Wiener filter bank.

\section{INTRODUCTION}

$\mathbf{T}$ HERE HAS been considerable research done on the signal reconstruction (deconvolution) of a stationary signal in a time-invariant channel [7], [9], [18]-[20]. In the past, the signal transmission system was modeled as a convolution between the input signal and the impulse response of the channel corrupted by noise. A Wiener filter or Kalman filter was employed to treat the deconvolution problem. Recently, the problem of reconstruction of nonstationary signals such as seismic data, acoustic signals, mechanical vibration, wireless communication, cyclostationary signals, radar and sonar signals, etc., has attracted the attention of signal processing researchers. Short-time Fourier transform, Gabor expansion, and wavelet transform techniques have been studied to analyze and synthesize these nonstationary signal processing. These techniques are based on so-called linear time-frequency (or time-scale) representations [4], [5], [8], [10]-[12], [14], [23].

In the conventional methods [11], one nonstationary signal is separated from other multicomponent nonstationary signals by the conventional mask technique in the time-frequency

Manuscript received July 11, 1996; revised March 19, 1998. This work was supported by National Science Council under Contract NSC 84-2213-007-073. The associate editor coordinating the review of this paper and approving it for publication was Prof. Moeness Amin.

The authors are with the Department of Electrical Engineering, National Tsing Hua University, Hsin-Chu, Taiwan, R.O.C.

Publisher Item Identifier S 1053-587X(98)08704-2. domain. In this situation, the spectrograms of these multicomponent signals cannot overlap in the time-frequency domain, or their performance will be deteriorated. Therefore, their applications are limited to some nonstationary signals. Hence, it is more appealing to find an optimal reconstruction (or deconvolution) to achieve optimal signal separation of multicomponent nonstationary signals with any kind of spectrogram in the time-frequency domain.

Recently, the multipath fading channel has been widely used to model the slowly time-varying channel in signal transmission, especially in sonar, radar, acoustics, seismic data processing, bioengineering, and oceanography. At present, it is still not easy to efficiently treat the signal reconstruction problem of a nonstationary signal transmitted through the multipath fading channel under nonstationary noise. Adaptive filtering algorithms have been developed to estimate the channel coefficients to update the reconstruction filter. However, they need a large number of computations to update the parameters via the adaptive algorithm in every update cycle. At present, the tracking ability of adaptive algorithms under a fading channel and a nonstationary signal and noise is still questionable. Furthermore, they need an input signal to train the update law. Hence, the way to design a deconvolution filter for a nonstationary (or time-varying) signal transmitted through a multipath fading channel with nonstationary noise is difficult but important work. To the best of our knowledge, there is still no good way to treat the deconvolution problem of a nonstationary signal transmitted through a multipath fading channel with nonstationary noise.

In this study, we solve the deconvolution filtering problem of nonstationary signal transmission through multipath fading and a noisy channel with the aid of bilinear ambiguity function (AF) techniques. The multipath fading channel has been successfully used in modeling slowly time-varying transmission systems.

Although linearity is a desirable property in a time-frequency representation, a quadratic structure provides an intuitively reasonable representation when we want to interpret a time-frequency response as a time-frequency energy distribution or instantaneous power spectrum. The reason for this is that it is a quadratic signal representation. An "energetic" time-frequency representation seeks to combine the concepts of instantaneous power $P_{x}(t)=|x(t)|^{2}$ and spectral energy density $P_{x}(f)=|X(f)|^{2}$. Apart from the "energetic" interpretation of a quadratic time-frequency rep- 
resentation, another possible interpretation uses a correlation function and spectral correlation function, both of which are, again, quadratic signal representations [4], [10], [14], [22], etc.

The AF is a quadratic time-frequency representation; it can be interpreted as a joint time-frequency correlation representation of a nonstationary signal. The AF and its square magnitude (the ambiguity surface or AS) have been extensively used in the fields of radar, sonar, radio astronomy, communications, and optics. In radar systems, the problem is the estimation of the distance and velocity of a moving target, where the distance and velocity correspond to the range parameter and the Doppler shift parameter, respectively. AF's and AS's have been used as analysis tools for the selection of radar waveforms. The AF has been applied to the design and evaluation of the performance of a large variety of radar signals including chirp and other FM signals [12], [14].

Recently, the AF has been used to perform time-varying filtering and multicomponent signal separation [10], [11]. Until now, research has been primarily devoted to the analysis and synthesis of AF in nonstationary signals. In this study, AF is employed in the time-frequency analysis and synthesis of a nonstationary signal transmission system in a multipath Rayleigh fading channel. The multipath Rayleigh fading channel has been successfully used in modeling slowly timevarying transmission systems. Therefore, the reconstruction of a nonstationary signal in a multipath fading channel is an important design topic in digital transmission systems. Based on time-frequency domain analysis and synthesis of the transmission system via AF transformation, an optimal signal deconvolution filter is designed. In other words, we focus our efforts on the solution of the optimal signal deconvolution filter design problem in nonstationary signal transmission systems.

The optimal time-frequency deconvolution filter consists of an AF filter bank and a Wiener filter bank, which is embedded in the AF filter bank (see Fig. 5). At the beginning, an analysis filter bank based on AF is developed to obtain the ambiguity (correlation) function of the received signal in the time-frequency domain. In each subband, the signal processing ambiguity (correlation) function is dependent on frequency only. Therefore, based on the calculus of variations and spectral factorization techniques, a Wiener filter bank is constructed to estimate the ambiguity (correlation) function of the transmitted signal from the ambiguity function of the received signal in each subband. The estimated ambiguity function of the transmitted signal in each subband is sent to the AF synthesis filter bank for transformation back to the time domain. Therefore, the design procedure of the proposed time-frequency deconvolution filter is divided into three stages. In the first stage, an AF analysis filter bank is constructed. In the second stage, a Wiener filter bank is developed to achieve the optimal signal reconstruction in the time-frequency domain. In the third stage, an AF synthesis filter bank is constructed to transform the estimated AF of the input signal in the time-frequency domain back to time domain. In this study, the channel noise is not restricted to be white Gaussian; it may be nonstationary or time-varying.

Finally, two simulation examples (one with optimal signal reconstruction and another with optimal separation) are

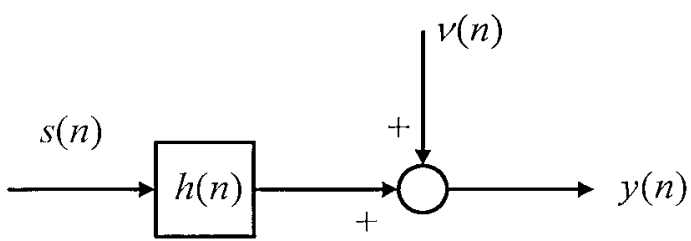

Fig. 1. System representation scheme.

given to illustrate the design procedure and demonstrate the signal reconstruction performance of the proposed optimal time-frequency deconvolution filter. The simulation results show that with the aid of an AF-based analysis/synthesis filter bank, the conventional Wiener filter bank design can be applied to solve the signal reconstruction problem of nonstationary signal transmission in a multipath Rayleigh fading channel.

\section{PROBLEM DESCRIPTION}

Consider a signal transmission system described by the discrete convolution system (see Fig. 1)

$$
\begin{aligned}
y(n) & =\sum_{l=0}^{L-1} \rho_{l}(n) s(n-l)+\nu(n) \\
& =H\left(q^{-1} ; n\right) s(n)+\nu(n)
\end{aligned}
$$

where

$s(n)$ transmitted signal;

$\rho_{l}(n)$ impulse response of transmission channel;

$\nu(n)$ channel noise;

$y(n)$ received signal.

In many practical applications, the time-varying transmission channels are described by the multipath fading model whose tap coefficients are Rayleigh distributed with p.d.f. $f\left(\rho_{l}\right)=$ $\frac{\rho_{l}}{\alpha_{l}^{2}} \exp \left(\frac{-\rho_{l}^{2}}{2 \alpha_{l}^{2}}\right)$ [21]. In this study, the channel operator is assumed to be the multipath fading form [21]

$$
H\left(q^{-1} ; n\right)=\sum_{l=0}^{L-1} \rho_{l}(n) q^{-l}
$$

where $q^{-1}$ denotes the backward shift operator defined by $q^{-1} s(n)=s(n-1)$, and $\rho_{l}(n), l=0,1, \ldots, L-1$ are the time-varying tap coefficients with $L$ being the number of different paths between the signal source and the destination.

We have the following assumptions:

1) The channel effects $\left\{\rho_{l}(n)\right\}$ in each path is a wide-sense stationary Rayleigh process, that is

$$
\begin{aligned}
E\left(\rho_{l}(n)\right) & =\sqrt{\frac{\pi}{2}} \alpha_{l}, \quad \forall n \\
E\left(\rho_{l}(n) \rho_{m}^{*}(k)\right) & =r_{\rho_{l}}(k-n) \delta[l-m]
\end{aligned}
$$

where $\delta[\cdot]$ is the unit impulse function.

2) The zero mean discrete-time process $\{\nu(n)\}$ is the additive noise process independent of the transmitted signal such that $E[\nu(n) \nu(n+k)]=\sigma_{\nu}^{2}(n, k)$, and $E\left[\nu\left(n_{1}\right) s\left(n_{2}\right)\right]=0$ for all $n_{1}, n_{2}$. 
Our signal reconstruction design problem is to reconstruct the input sequence $\{s(n)\}$ from the received data sequence $\{y(n)\}$ in the signal transmission system in (1). Since the input signal $s(n)$ and the channel noise are nonstationary (or time-varying) and the channel $H\left(q^{-1} ; n\right)$ is multipath fading, it is not easy to use a conventional filter, for example, a Wiener filter or Kalman filter, to reconstruct the transmitted sequence $\{s(n)\}$ from the received signal sequence $\{y(n)\}$. In the past few decades, the analysis and synthesis of nonstationary signals or time-varying systems using AF has been shown to be efficient. Therefore, it is suitable to employ AF techniques to treat the signal reconstruction problem of the nonstationary transmission system in (1). In this study, an AF analysis filter bank is first developed to transform the transmission system in (1) into an ambiguity system in time-frequency domain. Then, in each subband of the AF filter bank, a conventional Wiener filter is designed to optimally reconstruct the AF of the transmitted signal in the time-frequency domain. Finally, an AF synthesis filter bank is developed to transform the estimated AF of the input signal in the time-frequency domain back to the transmitted sequence $\hat{s}(n)$ (see Fig. 5). Before we discuss the design of an optimal Wiener filter bank for signal reconstruction, we will develop the design of an AF filter bank in the following section.

Remark: If the transmission system in (1) is free of channel, i.e., $h(n)=1$ such that $y(n)=s(n)+\nu(n)$ for all $n$, and $s(n)$ and $\nu(n)$ are all nonstationary or time-varying, then the optimal signal reconstruction problem becomes an optimal signal separation problem, i.e., to separate $s(n)$ from the multicomponent signal $y(n)=s(n)+\nu(n)$. This problem is an important research topic in time-frequency filter design, especially for the case with their representations overlapping in time-frequency domain.

\section{AF FILTER BANK DESIGN}

In this study, the AF filter bank plays a crucial role in the design of the optimal time-frequency deconvolution filter of a nonstationary transmission system. Before further discussion of this filter, the design of an AF filter bank is first considered. The so-called AF filter bank consists of an AF analysis filter bank and an AF synthesis filter bank. A brief review of the AF of a nonstationary signal, the design of an AF analysis filter bank, and an AF synthesis filter bank are discussed, respectively, in the following three subsections.

\section{A. A Brief Review of $A F$}

The AF used throughout this paper of a discrete-time stochastic process is defined as [1], [3]-[6], [12], [23]

$$
\begin{aligned}
\operatorname{AF}_{f, g}(k, w) & =\sum_{n=-\infty}^{\infty} E\left(f(n) g^{*}(n+k)\right) e^{-j w n} \\
& =\sum_{n=-\infty}^{\infty} R_{f, g}(n, k) e^{-j w n}
\end{aligned}
$$

where $E$ denotes the expectation operator, $j=\sqrt{-1}$ and $w \in[-\pi, \pi], k=0,1,2, \ldots$, and the superscript $*$ denotes the complex transpose. $\mathrm{AF}_{f, g}(k, w)$ can be considered to be a discrete Fourier transform of the cross-correlation function $R_{f, g}(n, k)$ of two random variables $f(n)$ and $g^{*}(n+k)$.

Remark: For deterministic function $f$ and $g$ [27], $R_{f, g}$ $(k, n)=f(n) g^{*}(k+n)$.

Similarly, the auto-AF of a discrete time sequence $\{f(n)\}$ is given by

$$
\begin{aligned}
\operatorname{AF}_{f, f}(k, w) & =\sum_{n=-\infty}^{\infty} E\left(f(n) f^{*}(n+k)\right) e^{-j w n} \\
& =\sum_{n=-\infty}^{\infty} R_{f, f}(n, k) e^{-j w n}
\end{aligned}
$$

Some properties of the AF, which are useful for the design of AF analysis/synthesis filter banks, are given as the following [4], [12], [23].

P1) Convolution in Time:

If the signal $z(n)$ is obtained by

$$
z(n)=x(n) *_{n} y(n)
$$

then we have

$$
\mathrm{AF}_{z, z}(k, w)=\sum_{m} \operatorname{AF}_{x, x}(m, w) \mathrm{AF}_{y, y}(k-m, w)
$$

P2) Finite Support:

$$
\begin{aligned}
& \text { If } f(n)=0 \text { for }|n|>N \text {, then } \\
& \qquad \mathrm{AF}_{f, f}(k, w)=0
\end{aligned}
$$

for $|k|>2 N$.

$$
\begin{array}{r}
\text { If } F(w)=0 \text { for }|w|>W \text {, then } \\
\operatorname{AF}_{F, F}(k, w)=0
\end{array}
$$

for $|w|>2 W$.

\section{P3) Bilinear Property:}

From the definition of AF in (3), we have

$$
\begin{aligned}
\mathrm{AF}_{f_{1}+f_{2}, g_{1}+g_{2}}(k, w) & =\mathrm{AF}_{f_{1}, g_{1}}(k, w)+\mathrm{AF}_{f_{2}, g_{2}}(k, w) \\
& +\mathrm{AF}_{f_{2}, g_{1}}(k, w)+\mathrm{AF}_{f_{1}, g_{2}}(k, w) .
\end{aligned}
$$

\section{B. AF Analysis Filter Bank}

The properties of the AF discussed in Section III-A form the basis for the use of AF in the AF filter bank design in this study. For practical reasons, in this study, we are dealing with the AF not only in the discrete time domain but in the discrete frequency domain as well. Further, only a finite number of sample sequence is used. The fast Fourier transform (FFT) technique is well suited to treat the fast computation problem of the discrete AF transformation. In this situation, the finite sample sequence can be viewed as an infinite sequence with finite support $[0, N-1]$. Thus, a pseudo AF is defined with a finite window to mask the data sequence in the $\mathrm{AF}$ transformation

$$
\mathrm{AF}_{f, f}(k, w)=\sum_{n=0}^{N-1} E\left(f(n) f^{*}(n+k)\right) e^{-j w n}
$$




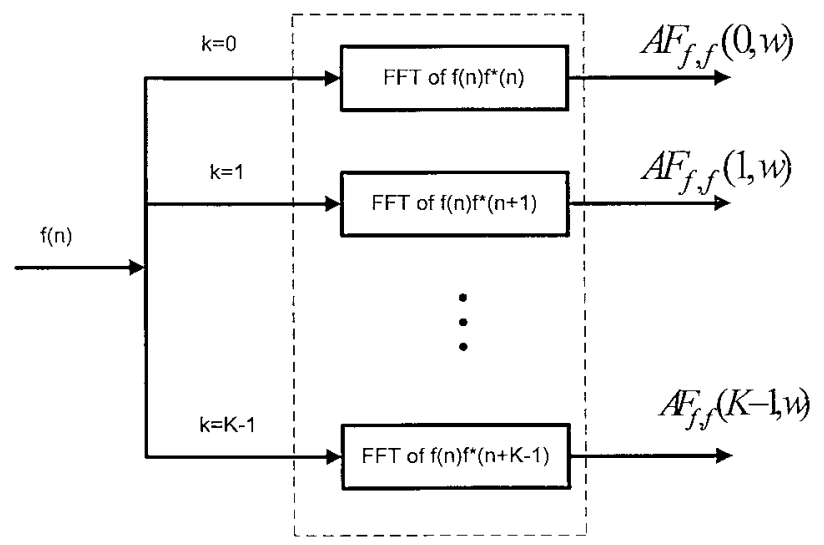

Fig. 2. AF analysis filter bank.

where $k=0,1,2, \ldots, K-1$, and $w$ denotes the continuous frequency variable in $[-\pi, \pi]$. We now sample the frequency variable as

$$
w_{m}=\frac{2 \pi m}{N}, \quad m=0,1, \ldots, N-1
$$

and then substitute (11) into (10) to obtain the AF as

$$
\mathrm{AF}_{f, f}(k, m)=\sum_{n=0}^{N-1} R_{f, f}(n, k) e^{-j m n 2 \pi / N}
$$

where $k=0,1,2, \ldots, K-1, m=0,1,2, \ldots, N-1$.

According to the above $\mathrm{AF}$, the $\mathrm{AF}$ of the discrete sequence $f(n)$ in (12) can be considered to be the discrete Fourier transform of $R_{f, f}(n, k)$ for all $k=0,1,2, \ldots, K-1$ and $n=0,1,2, \ldots, N-1$. Therefore, the FFT technique can be employed for fast computation of the AF transformation in (12). In this situation, the $\mathrm{AF}$ of the discrete sequence $f(n)$ in (12) can be obtained in parallel by an analysis filter bank, as shown in Fig. 2. In each subband of the AF analysis filter bank in Fig. 2, $\operatorname{AF}_{f, f}(k, w)$ is only a function of the frequency $w$ for $k=0,1,2, \ldots K-1$. Thus, the frequency domain optimal estimation technique, i.e., the Wiener filtering, can be employed in each subband to estimate the AF of the transmitted signal.

Remark: The AF in (12) is more useful than the AF in (4) from the point of view of practical implementation. In this study, an AF analysis filter bank is implemented via the AF in Fig. 2 to perform the AF transformation of nonstationary signals.

After the sampled nonstationary signal $y(n)$ in (1) is transformed by the $\mathrm{AF}$ analysis filter bank into an $\mathrm{AF}$ in the time-frequency domain, a Wiener filter bank will be used to estimate the $\mathrm{AF}$ of the transmitted signal $s(n)$ in the time-frequency domain. Thus, an $\mathrm{AF}$ synthesis filter bank must be employed to transform the estimated $\mathrm{AF}$ of the transmitted signal in time-frequency domain back to the estimation of the transmitted sequence $\hat{s}(n)$ in the time domain (see Fig. 5). For the convenience of discussion, the design of the AF synthesis filter bank will be described in the Section III-C, and the design of the Wiener filter bank will be developed in Section IV.

\section{AF Synthesis Filter Bank}

In this study, the AF synthesis filter bank is used to transform the estimated AF of the transmitted signal into the time-frequency domain, which is obtained by the Wiener filter bank, back to the estimated transmitted signal $\hat{s}(n)$ in the time domain.

The AF synthesis is approached by minimizing the integrated square error between an arbitrary desired AF and a realizable AF [23]-[25], whose corresponding signal in the time domain can be easily obtained. This is an optimal approximation problem in the time-frequency domain, and the solution is proposed via an orthonormal basis method over the time-frequency plane. The mean square error MSE and the corresponding signal are determined through an eigenvalue approximation either by complex auto-AF or by complex cross-AF.

The following derivation of an AF synthesis filter bank is based on the result in [23]. In the derivation, given a desired ambiguity function $\operatorname{AF}_{x, x}(n, w)$, we set out to find a realizable ambiguity function $\theta(n, w)$, which can be easily transformed back to a time function $x(n)$, to optimally approximate $\operatorname{AF}_{x, x}(n, w)$. The details are as follows.

Suppose the signal $x(n)$ can be decomposed by the orthogonal basis function set $\left\{\phi_{i}\right\}$

$$
x(n)=\sum_{i} a_{i} \phi_{i}(n) .
$$

Then, the realizable AF corresponding to $x(n)$ is of the form

$$
\theta(k, w)=\sum_{i} \sum_{l} \sum_{n} a_{i} a_{l}^{*} R_{\phi_{i}, \phi_{l}}(n, k) e^{-j w n} .
$$

To specify the parameters $a_{i}, i=1,2, \ldots$ of the realizable AF $\theta(k, w)$ to approximate the given $\operatorname{AF}_{x, x}(k, w)$, the MSE method

$$
\epsilon=\frac{1}{2 \pi} \iint\left|\operatorname{AF}_{x, x}(\tau, w)-\theta(\tau, w)\right|^{2} d \tau d w
$$

is used.

From [23], we can rewrite the above continuous equation in the discrete time and discrete frequency form as

$$
\epsilon=\frac{1}{N} \sum_{n=0}^{2 K-1} \sum_{m=0}^{N-1}\left|\mathrm{AF}_{x, x}(n, m)-\theta(n, m)\right|^{2}
$$

where

$$
\theta(n, m)=\sum_{i} \sum_{l} a_{i} a_{l}^{*} \Phi_{i, l}(n, m)
$$

and

$$
\Phi_{i, l}=\sum_{k} R_{\phi_{i}, \phi_{l}}(n, k) e^{-j m k 2 \pi / N} .
$$

By P2), the sampled signal basis functions are assumed to vanish outside the interval $0 \leq k \leq K-1$ and to be orthonormal, i.e.,

$$
\phi_{i}(k)=0
$$


for $k<0$ or $k>K$ and

$$
\sum_{k=1}^{K} \phi_{i}(k) \phi_{l}^{*}(k)=\delta_{i l} .
$$

The main work of the AF synthesis is to specify the parameters $a_{i}, i=1,2, \ldots$ in (13) to minimize the MSE in (16).

Expanding the equation in (16) gives

$$
\begin{aligned}
\epsilon= & \left\|\mathrm{AF}_{x, x}\right\|^{2}+\sum_{i} \sum_{l}\left|a_{i} a_{l}^{*}\right|^{2}-\sum_{i} \sum_{l} a_{i} a_{l}^{*}\left(\mathrm{AF}_{x, x}, \Phi_{i, l}\right) \\
& -\sum_{i} \sum_{l} a_{i}^{*} a_{l}\left(\Phi_{i, l}, \mathrm{AF}_{x, x}\right)
\end{aligned}
$$

where

$$
\left\|\mathrm{AF}_{x, x}\right\|^{2}=\frac{1}{N} \sum_{n=0}^{2 K-1} \sum_{m=0}^{N-1}\left|\mathrm{AF}_{x, x}(n, m)\right|^{2}
$$

and

$$
\left(\mathrm{AF}_{x, x}, \Phi_{i, l}\right)=\frac{1}{N} \sum_{n=0}^{2 K-1} \sum_{m=0}^{N-1} \operatorname{AF}_{x, x}(n, m) \Phi_{i, l}(n, m) .
$$

For the convenience of notation, we denote

$$
B_{i, l}:=\left(\mathrm{AF}_{x, x}, \Phi_{i, l}\right) .
$$

By some rearrangements, (19) can be expressed as

$$
\epsilon=\left\|\mathrm{AF}_{x, x}\right\|^{2}+\left[\mathbf{a}^{* T} \mathbf{a}\right]^{2}-\mathbf{a}^{* T}\left(B+B^{* T}\right) \mathbf{a}
$$

where $\mathbf{a}=\left[a_{0}, a_{1}, \ldots\right]^{T}, T$ denotes the transpose, and $B$ is a matrix whose entries are given in (22).

The optimal a, which achieves the minimum in (23), must satisfy [23]

$$
\left(2 \mathbf{a}^{* T} \mathbf{a}\right) \mathbf{a}-\left(B+B^{* T}\right) \mathbf{a}=0
$$

i.e.,

$$
\left(B+B^{* T}\right) \mathbf{a}=2 E \mathbf{a}
$$

where

$$
E=\mathbf{a}^{* T} \mathbf{a}=\sum_{i}\left|a_{i}\right|^{2} .
$$

The equation in (25) is viewed as the desired extreme condition. By inserting (25) into (23), we obtain the optimal approximation error as

$$
\epsilon=\left\|\mathrm{AF}_{x, x}\right\|^{2}-E^{2} .
$$

\section{Remarks:}

1) In this study, the sinusoid functions are chosen as basis functions $\phi_{i}(n)$ in (13).

2) Equations (25)-(27) completely specify the solution for the minimum approximation error. Since $\epsilon$ is nonnegative, a minimum approximation error is attained when $E^{2}$ is a maximum in (27). Therefore, from (25), it is seen that $2 \mathrm{E}$ must be the largest positive eigenvalue of $\left(B+B^{* T}\right)$, and $\mathbf{a}$ is the corresponding eigenvector, whose magnitude is adjusted to satisfy the energy condition $\mathbf{a}^{* T} \mathbf{a}=E$. Then, $\mathbf{a}$ represents the desired signal weights in terms of the previously chosen basis functions.
After $\hat{\mathbf{a}}$ is solved from the eigenvector of $\left(B+B^{* T}\right)$ corresponding to the largest eigenvalue in (25), the signal $\hat{x}(n)$ corresponding to the realizable $\theta(k, w)$, which optimally approximates a given $\operatorname{AF}_{x, x}(n, m)$, is obtained as

$$
\hat{x}(n)=\sum_{i} \hat{a}_{i} \phi_{i}(n) .
$$

Therefore, from (21), (22), (25), and (28), an AF synthesis filter bank is constructed as Fig. 3. In Fig. 3, a denotes the reconstruction coefficient vector $\left[\hat{a}_{0}, \hat{a}_{1}, \ldots, \hat{a}_{N-1}\right]$, and $\Psi$ represents the basis functions $\left[\phi_{0}, \phi_{1}, \ldots, \phi_{N-1}\right]$. In our design, the AF synthesis filter bank transforms the estimated $\mathrm{AF}$ of the transmitted signal, which is obtained by Wiener filter bank in the previous stage (see Fig. 5) back to the time domain to reconstruct the transmitted signal.

\section{WiENER Filter BANK Design}

The design of Wiener filter bank is central to the proposed signal deconvolution filter. In this study, the Wiener filter determines the optimal AF reconstruction of the transmitted signal in each subband in the frequency domain. Before derivation of the Wiener filter bank, the AF analysis of the transmission system of (1) in the time-frequency domain is discussed.

By property P1) and P2), the AF of the signal transmission system in (1) is of the form

$$
\begin{aligned}
\mathrm{AF}_{y, y}(k, w)= & \sum_{m}\left[\mathrm{AF}_{h, h}(k-m, w) \mathrm{AF}_{s, s}(m, w)\right] \\
& +\mathrm{AF}_{\nu, \nu}(k, w)+\mathrm{AF}_{\nu, s * h}(k, w) \\
& +\mathrm{AF}_{s * h, \nu}(k, w)
\end{aligned}
$$

for $k=0,1, \ldots K-1, w \in[-\pi, \pi]$, where $K$ is the number of subbands, and $\operatorname{AF}_{\nu, \nu}(k, w)$ is the $\mathrm{AF}$ of the noise $\nu(n)$, i.e., the AF of the signal $y(n)$ at the output of the $k$ th subband of the AF analysis filter bank in Fig. 5. The last two terms denote the cross ambiguity functions.

From (2), we have

$$
\begin{aligned}
\mathrm{AF}_{h, h}(k, w) & =\sum_{n} \sum_{l} r_{\rho_{l}}(k) e^{-j w n} \\
\mathrm{AF}_{\nu, \nu}(k, w) & =\sum_{n} R_{\nu, \nu}(n, k) e^{-j w n} \\
& =\sum_{n} \sigma_{\nu}^{2}(n, k) e^{-j w n} .
\end{aligned}
$$

In addition, $\nu(n)$ is independent of the output of the transmitted signal $s(n) * h(n)=\sum_{l=0}^{L-1} \rho_{l}(n) s(n-l)$, and we have

$$
\begin{aligned}
R_{\nu, s * h}(n, k) & =E\left[\nu(n) \sum_{l=0}^{L-1}\left(\rho_{l}(n) s(n-l)\right)^{*}\right] \\
& =E[\nu(n)] E\left[\sum_{l} \rho_{l}^{*}(n) s(n-l)^{*}\right]=0
\end{aligned}
$$

for all $n$; hence

$$
\mathrm{AF}_{\nu, s * h}(k, w)=0 .
$$




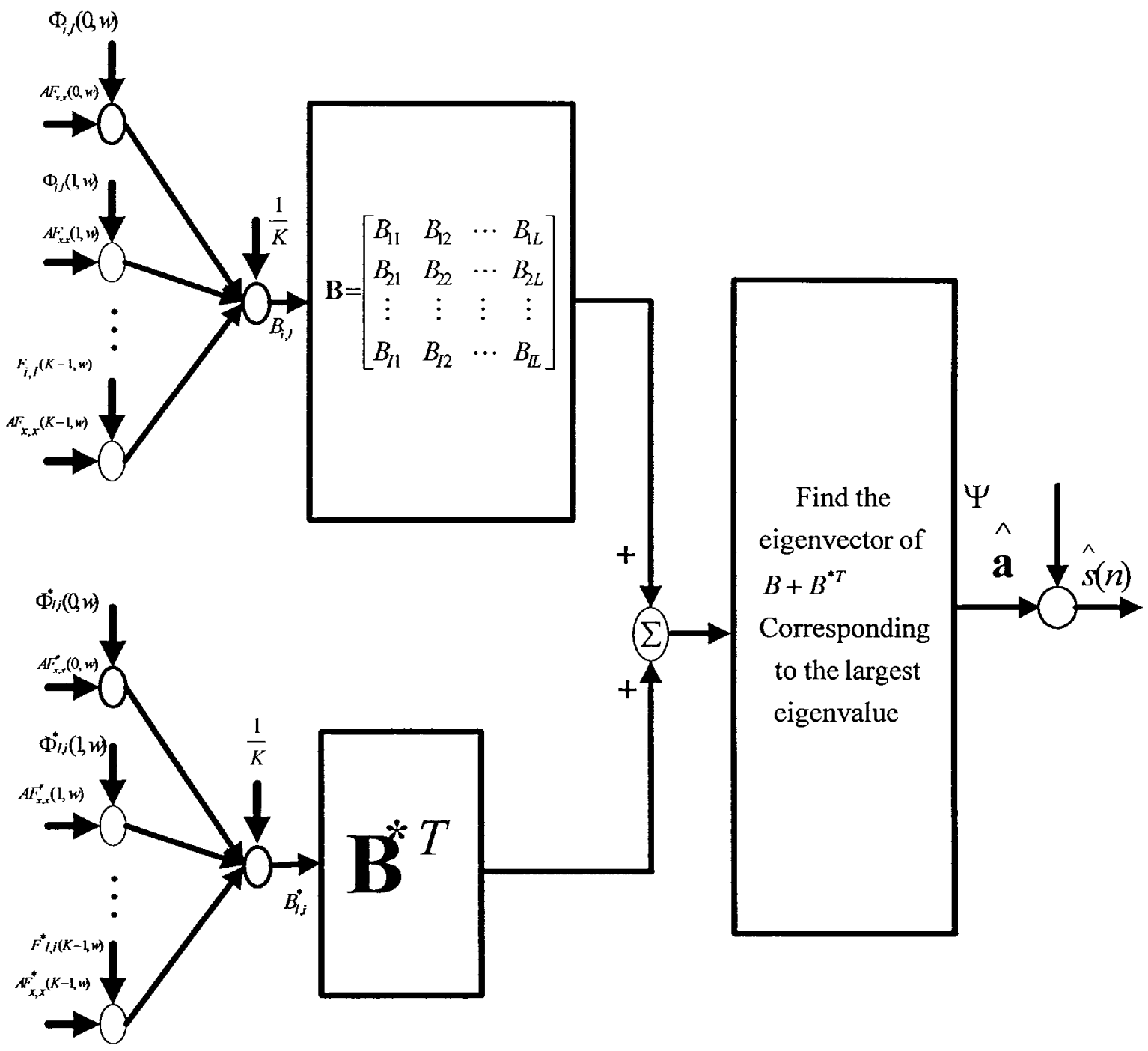

Fig. 3. AF synthesis filter bank.

Similarly, $\mathrm{AF}_{s * h, \nu}(k, w)=0$. Therefore, (29) can be rewritten as

$$
\begin{aligned}
\operatorname{AF}_{y, y}(k, w)= & \sum_{m}\left(\sum_{n} \sum_{l} r_{\rho_{l}}(k-m) e^{-j w n}\right) \mathrm{AF}_{s, s}(m, w) \\
& +\sum_{n} \sigma_{\nu}^{2}(n, k) e^{-j w n} \\
= & \prod(k, w)+\mathbf{D}(k, w)
\end{aligned}
$$

where $\prod(k, w):=\sum_{m}\left(\sum_{n} \sum_{l} r_{\rho_{l}}(k-m) e^{-j w n}\right)$ $\mathrm{AF}_{s, s}(m, w)$, and $\mathbf{D}(k, w):=\sum_{n} \sigma_{\nu}^{2}(n, k) e^{-j w n}$. In the analysis of the previous sections, our main problem is to eliminate the noise ambiguity function $\operatorname{AF}_{\nu, \nu}(k, w)$ and remove the channel effect from $\mathrm{AF}_{y, y}(k, w)$ to estimate the $\mathrm{AF}$ of the transmitted signal $\mathrm{AF}_{s, s}(k, w)$. In the $k$ th subband, $\mathrm{AF}_{y, y}(k, w)$ represents a spectral density of a stationary signal. Therefore, the Wiener filtering technique is employed to achieve an optimal estimation of $\mathrm{AF}_{s, s}(k, w)$ from $\mathrm{AF}_{y, y}(k, w)$. The estimation for the $k$ th subband of the AF filter bank is explicitly formulated as in Fig. 4. Referring to the signal reconstruction via Wiener filter in Fig. 4, given

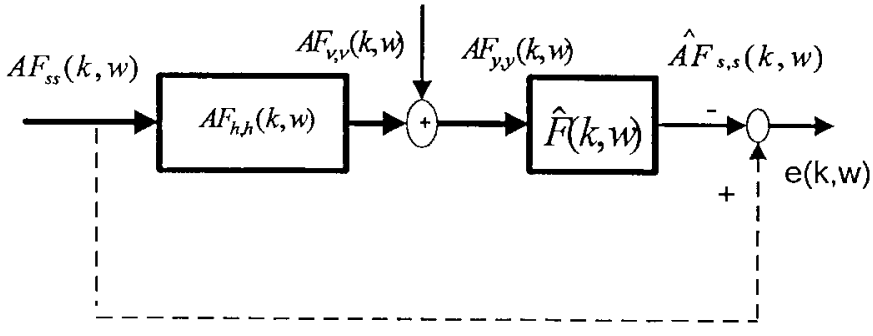

Fig. 4. Reconstruction problem of the $k$ th band.

the received $\operatorname{AF}_{y, y}(k, w)$ at the output of the $k$ th subband of the AF analysis filter bank, which is the AF of the input signal $\mathrm{AF}_{s, s}(k, w)$ convolved with the $\mathrm{AF}$ of the channel $\mathrm{AF}_{h, h}(k, w)$ with respect to $k$ and corrupted by the $\mathrm{AF}$ of the noise $\mathrm{AF}_{\nu, \nu}(k, w)$ [see (29) or (34)], the reconstruction problem at the $k$ th subband lies in how to specify a filter $F(k, w)$ to optimally estimate $\mathrm{AF}_{s, s}(k, w)$ from the received $\mathrm{AF}_{y, y}(k, w)$.

The estimation error $e(k, w)$ of the Wiener filter $F(k, w)$ in the $k$ th subband of the AF filter bank can be expressed as 
(see Fig. 4)

$$
\begin{aligned}
e(k, w)= & \mathrm{AF}_{s, s}(k, w)-F(k, w) \mathrm{AF}_{y, y}(k, w) \\
= & \mathrm{AF}_{s, s}(k, w)-F(k, w) \prod(k, w) \\
& -F(k, w) \mathbf{D}(k, w) .
\end{aligned}
$$

The current problem lies in finding an optimal estimator $\hat{F}(k, w)$ in the $k$ th subband such that the MSE

$$
J_{k}=\frac{1}{2 \pi} \int_{-\pi}^{\pi}\left\{e(k, w) e^{*}(k, w)\right\} d w
$$

is minimized. We can make the substitution $z=e^{j w}$ to express the above cost equation as [19]

$$
J_{k}=\frac{1}{2 \pi j} \oint_{|z|=1}\left\{e(k, z) e^{*}(k, z)\right\} \frac{d z}{z} .
$$

The MSE of the cost function $J_{k}$ in the $k$ th subband is

$$
\begin{aligned}
J_{k}= & \frac{1}{2 \pi j} \oint_{|z|=1} J_{k}^{(0)}(z)-J_{k}^{(1)}(z) F^{*}(k, z) \\
& -J_{k}^{(1)^{*}}(z) F(k, z)+J_{k}^{(2)}(z) F(k, z) F^{*}(k, z) \frac{d z}{z}
\end{aligned}
$$

where

$$
\begin{aligned}
J_{k}^{(0)}(z)= & \mathrm{AF}_{s, s}(k, z) \mathrm{AF}_{s, s}^{*}(k, z) \\
J_{k}^{(1)}(z)= & \mathrm{AF}_{s, s}(k, z) \prod^{*}(k, z)+\mathrm{AF}_{s, s}(k, z) \mathrm{D}^{*}(k, z) \\
J_{k}^{(2)}(z)= & \prod(k, z) \prod^{*}(k, z)+\prod^{*}(k, z) \mathbf{D}(k, z) \\
& +\mathrm{D}(k, z) \prod^{*}(k, z)+\mathrm{D}^{*}(k, z) \mathrm{D}(k, z)
\end{aligned}
$$

Then, our design problem in the $k$ th subband involves how to specify an optimal filter $\hat{F}(k, z)$ to minimize the above performance $J_{k}$. In the following, the calculus of variation technique in the $Z$ domain will be employed to treat the optimal causal stable filter design problem to estimate the AF of the input signal $s(n)$. Let $F(k, z)=\hat{F}(k, z)+\varepsilon \eta(z)$ be a candidate for the optimal filter, where $\hat{F}(k, z)$ denotes the Wiener filter to be derived, and $\eta(z)$ is any realizable function with all poles in $|z|<1 . \varepsilon$ is assumed to be an arbitrarily small real number [8], [15], [19], i.e., $F(k, z)$ is a realizable perturbation of the Wiener filter $\hat{F}(k, z)$. The MSE can be rewritten as

$$
\begin{aligned}
J_{k}= & \frac{1}{2 \pi j} \oint_{|z|=1} J_{k}^{(0)}-J_{k}^{(1)}(\hat{F}(k, z)+\varepsilon \eta(z))^{*} \\
& -J_{k}^{(1) *}(\hat{F}(k, z)+\varepsilon \eta(z))+J_{k}^{(2)}(\hat{F}(k, z) \\
& +\varepsilon \eta(z))(\hat{F}(k, z)+\varepsilon \eta(z))^{*} \frac{d z}{z}
\end{aligned}
$$

Based on the calculus of variation technique and symmetrical property, the minimun MSE (MMSE) must satisfy [8], [19]

$$
\begin{aligned}
\left.\frac{\partial J_{k}}{\partial \varepsilon}\right|_{\varepsilon=0} & =\frac{-2}{2 \pi j} \oint_{|z|=1}\left[\hat{F}(k, z) J_{k}^{(2)}(z)-J_{k}^{(1)}(z)\right] \eta^{*}(z) \frac{d z}{z} \\
& =0
\end{aligned}
$$

i.e., $\oint_{|z|=1}\left[\hat{F}(k, z) J_{k}^{(2)}(z)-J_{k}^{(1)}(z)\right] \eta^{*}(z) \frac{d z}{z}=0$ from the above equation. For the causal and stable realization of $\hat{F}(k, z)$, the spectral factorization technique is employed to treat the above optimal filter design problem. Perform the spectral factorization [15]

$$
\triangle_{k}^{*}(z) \triangle_{k}(z)=J_{k}^{(2)}(z)
$$

where the $\triangle_{k}(z)$ is free of poles and zeros in $|z| \geq 1$. Substitution of (41) into (40) yields

$$
\begin{aligned}
& \oint_{|z|=1}\left\{\hat{F}(k, z) \triangle_{k}(z) \triangle_{k}^{*}(z)-J_{k}^{(1)}(z)\right\} \eta^{*}(z) \frac{d z}{z} \\
& =\oint_{|z|=1}\left\{\hat{F}(k, z) \triangle_{k}(z)-\frac{J_{k}^{(1)}(z)}{\triangle_{k}^{*}(z)}\right\} \triangle_{k}^{*}(z) \eta^{*}(z) \frac{d z}{z} \\
& =0 .
\end{aligned}
$$

The term $\left\{g_{k}(z)\right\}:=\frac{J_{k}^{(1)}(z)}{\triangle_{k}^{*}(z)}$ is decomposed as

$$
\left\{g_{k}(z)\right\}=\left\{g_{k}(z)\right\}_{+}+\{g(z)\}_{-}
$$

where $\left\{g_{k}(z)\right\}_{+}$and $\left\{g_{k}(z)\right\}_{-}$denote the parts that are analytic outside and inside the unit circle, respectively. Substituting (43) into (42) yields

$$
\begin{gathered}
\oint_{|z|=1}\left[\hat{F}(k, z) \triangle_{k}(z)-\left\{g_{k}(z)\right\}_{+}\right] \eta^{*}(z) \triangle_{k}^{*}(z) \frac{d z}{z} \\
-\oint_{|z|=1} \hat{F}(k, z)\left\{g_{k}(z)\right\}_{-} \eta^{*}(z) \triangle_{k}^{*}(z) \frac{d z}{z}=0 .
\end{gathered}
$$

By Cauchy's theorem, the second term of the left-hand side in (44) is equal to zero since all of its poles are located in $|z| \geq 1$. Hence, we obtain

$\oint_{|z|=1}\left[\hat{F}(k, z) \triangle_{k}(z)-\left\{g_{k}(z)\right\}_{+}\right] \eta^{*}(z) \triangle_{k}^{*}(z) \frac{d z}{z}=0$.

Note that all of the poles of $\eta^{*}(z) \triangle_{k}^{*}(z)$ are in $|z| \geq 1$, and those of the term $\hat{F}(k, z) \triangle_{k}(z)-\left\{g_{k}(z)\right\}_{+}$are in $|z|<1$. By Cauchy's theorem, (45) holds (i.e., the integration around the unit circle must be zero) if and only if

$$
\hat{F}(k, z) \triangle_{k}(z)-\left\{g_{k}(z)\right\}_{+}=0 .
$$

The optimal Wiener filter $\hat{F}(k, z)$ in the $k$ th subband of the AF filter bank is therefore derived from the above equation as

$$
\hat{F}(k, z)=\frac{\left\{g_{k}(z)\right\}_{+}}{\triangle_{k}(z)} \quad k=0,1, \ldots, K-1 .
$$

The Wiener filter in the above (46) is a causal stable filter and can be realized by a recursive structure.

After the Wiener filter bank $\hat{F}(k, z), k=0,1, \ldots, K-1$ has been designed as in (46), it is implemented as shown in Fig. 5 to estimate the ambiguity function $\operatorname{AF}_{s, s}(k, w)$ of the transmitted signal in each subband from the received ambiguity function $\mathrm{AF}_{y, y}(k, w)$ for all $k=0,1, \ldots, K-1$. 


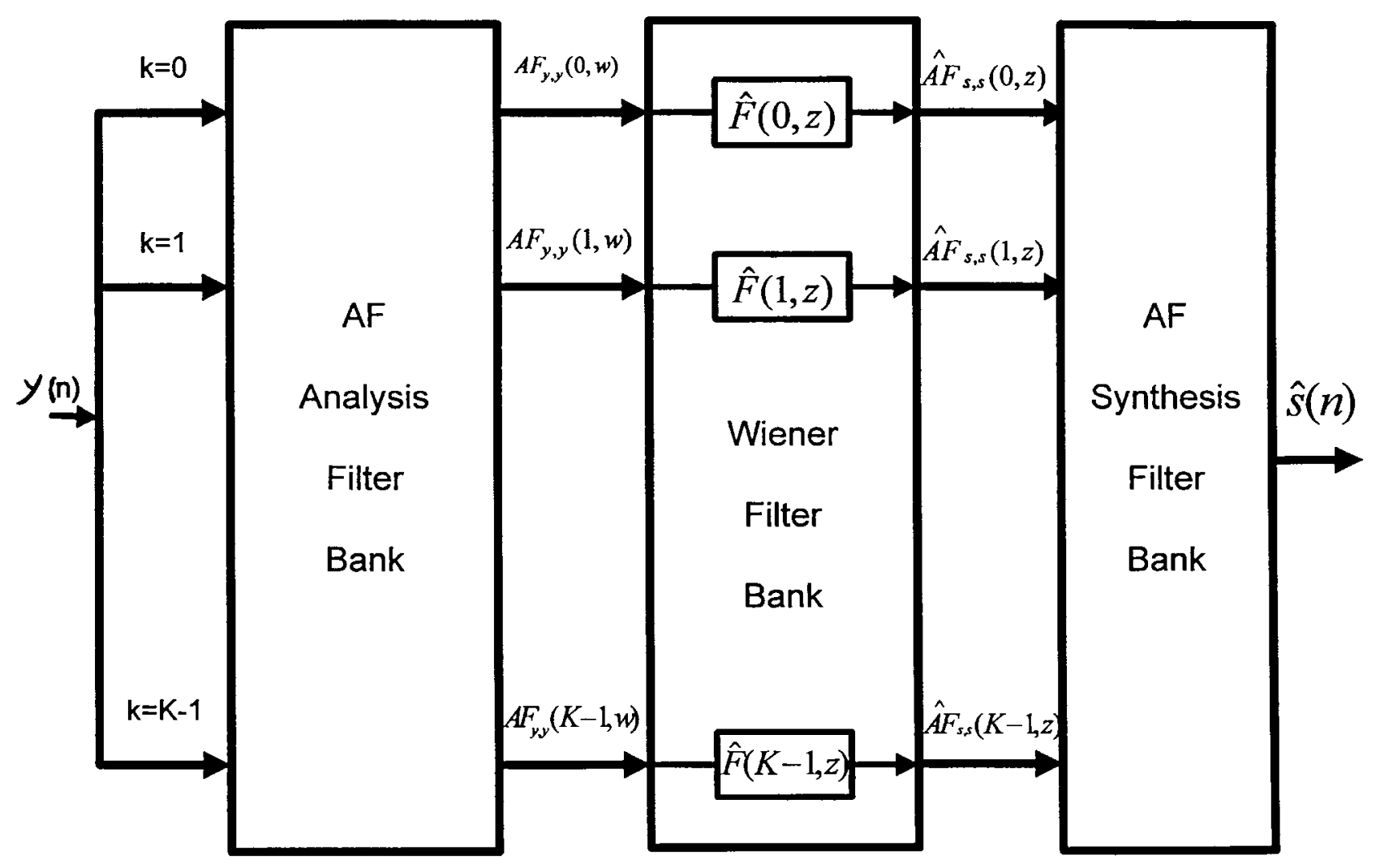

Fig. 5. AF filter bank-based optimal signal deconvolution filter.

Then, the estimated $\hat{\mathrm{AF}}_{s, s}(k, w)$, for $k=0,1, \ldots, K-1$ are sent to the AF synthesis filter bank to synthesize the estimated signal $\hat{s}(n)$.

Remark:

1) From (37) and (46), the minimum MSE (i.e., MMSE) $\hat{J}_{k}$ is also derived, under the proposed optimal filter, as

$$
\begin{aligned}
\hat{J}_{k}= & \frac{1}{2 \pi j} \oint_{|z|=1} J_{k}^{(0)}(z)-J_{k}^{(1)}(z) \hat{F}(k, z) \\
& -J_{k}^{(1) *}(z) \hat{F}^{*}(k, z)+J_{k}^{(2)}(z) \hat{F}(k, z) \hat{F}^{*}(k, z) \frac{d z}{z} .
\end{aligned}
$$

$\hat{J}_{k}$ can be easily calculated by Cauchy's integral formula or residue theorem.

2) If the system is free of channel, i.e., $h(n)=1$, and $s(n)$ and $v(n)$ are all nonstationary signals, then $y(n)=$ $s(n)+\nu(n)$ is a multicomponent nonstationary signal. In this situation, the proposed method becomes how to optimally separate $s(n)$ from $y(n)$, i.e., to solve the signal separation problem [14].

\section{Simulations}

In this section, two numerical examples of nonstationary or time-varying signal transmission through a multipath fading channel are given to illustrate the reconstruction performance of the proposed optimal time-frequency deconvolution filter. The main concern is with the reconstruction of the input signal. We define the reconstruction performance $\mathrm{SNR}_{r}:=$
$10 \log _{10}\left(\frac{\operatorname{Var} s(n)}{\operatorname{Var} e(n)}\right) \mathrm{dB}$. Based on $M_{1}=30$ realizations of input signals $\{s(n)\}$, each with $M_{2}=100$ Monte-Carlo simulations, the average reconstruction performance is indicated by the relationship

$$
\overline{\mathrm{SNR}_{r}}:=\frac{1}{M_{1} M_{2}} \sum_{i=1}^{M_{1}} \sum_{j=1}^{M_{2}} 10 \log _{10}\left(\frac{\operatorname{Var}\left(s_{i, j}(n)\right)}{\operatorname{Var}\left(e_{i, j}(n)\right)}\right) \mathrm{dB}
$$

where $e_{i, j}(n)=s_{i, j}(n)-\hat{s}_{i, j}(n)$ denotes the reconstruction error for the $i, j$ th realization. Another signal-to-noise ratio $\mathrm{SNR}_{s}$ is defined as the following for illustration of the input signal-to-noise ratio:

$$
\mathrm{SNR}_{s}:=10 \log _{10}\left(\frac{\operatorname{Var}(s(n))}{\operatorname{Var}(\nu(n))}\right) \mathrm{dB} .
$$

Example 1-Fractal Signal Transmission Case: The first example involves a fractional Brownian motion (FBM) signal in a fading channel corrupted by noise. There are two types of noises considered in this example, i.e., white Gaussian noise and jammer in case 1 and case 2 in the sequel, respectively. The channels are assumed to be the three-path fading channel

$$
H\left(q^{-1} ; n\right)=\rho_{0}+\rho_{1} q^{-1}+\rho_{2} q^{-2}
$$

where $\rho_{0}, \rho_{1}$, and $\rho_{2}$ are independent random variables with Rayleigh distributions with $\alpha_{0}=\sqrt{2}, \alpha_{1}=0.1$, and $\alpha_{2}=$ 0.02 , respectively. The FBM is a nonstationary stochastic 


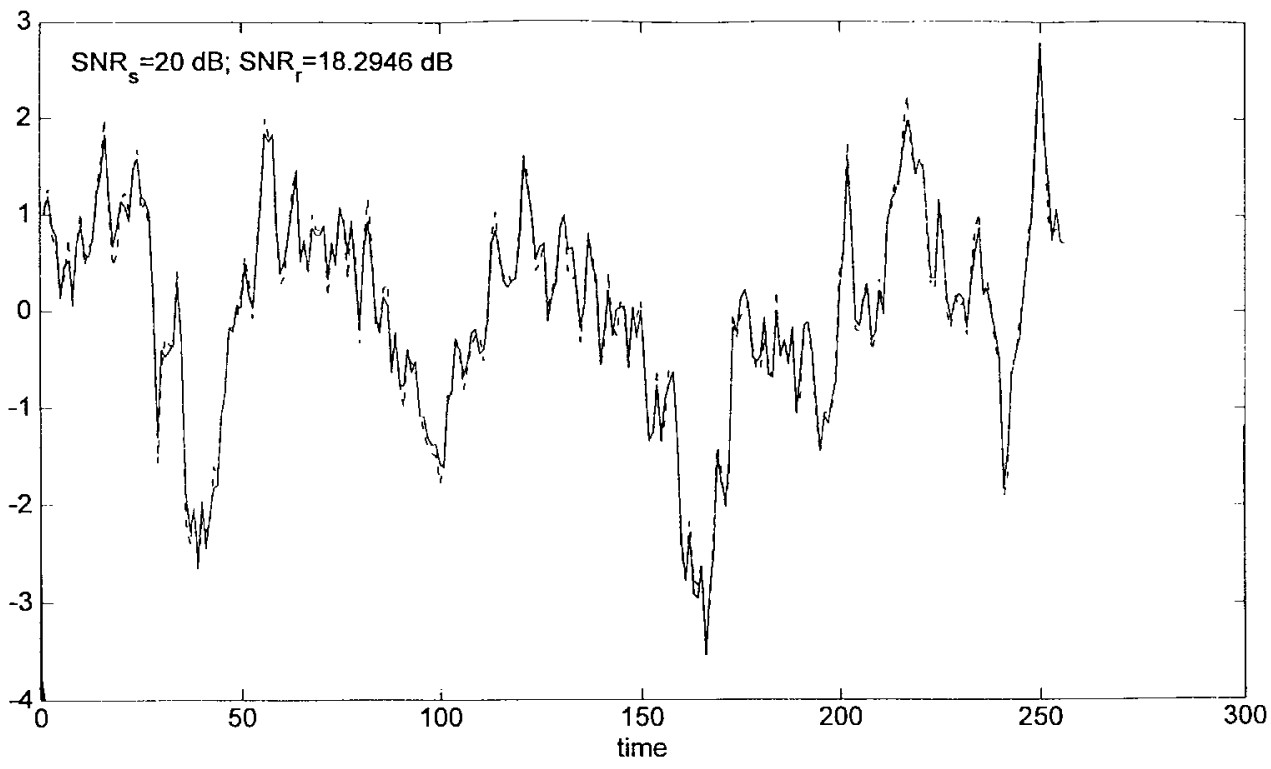

Fig. 6. Signal reconstruction in Example 1 with white Gaussian noise. $(s(n)$ : solid line; $\hat{s}(n)$ : dashed line).

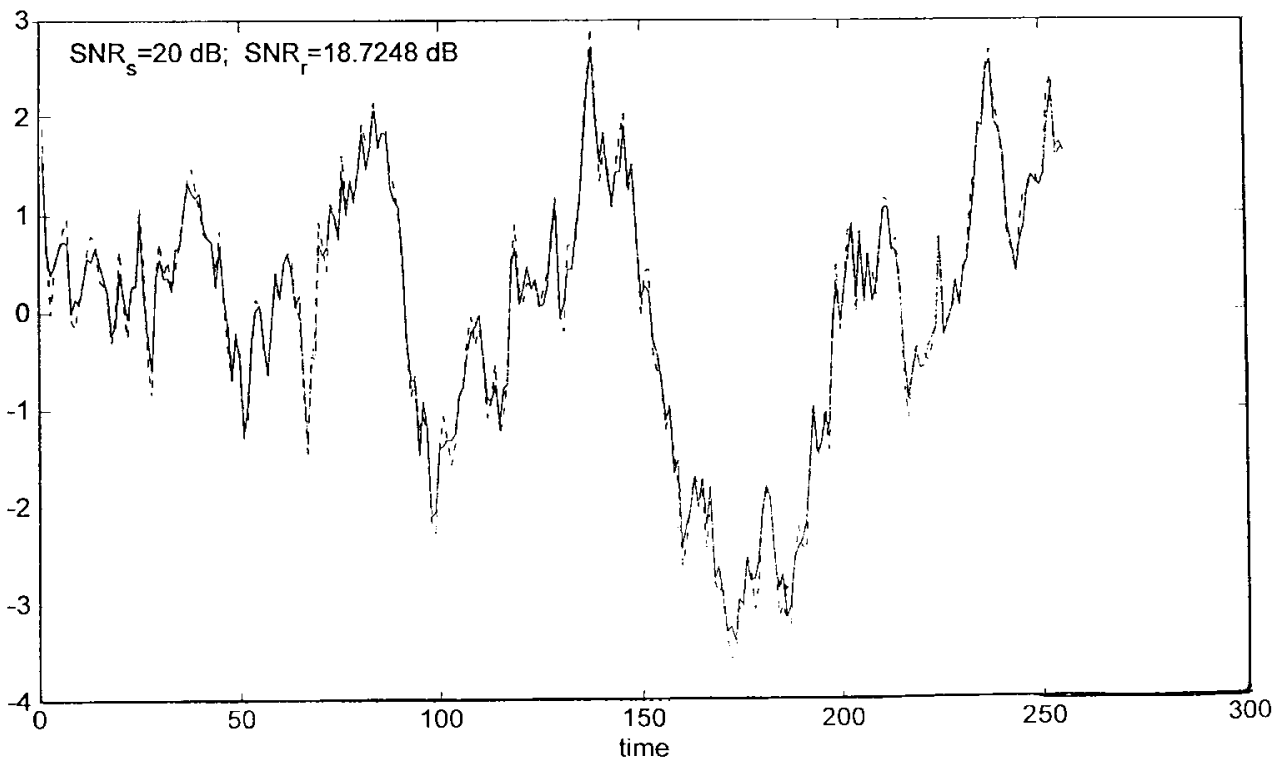

Fig. 7. Signal reconstruction in Example 1 with $\cos (n+.1913)+\sin (2 n)$ as the jammer. $(s(n)$ : solid line; $s(n)$ : dashed line).

process of the [13], [16]

$$
\begin{aligned}
B(0 ; H)= & 0 \\
B(t ; H)-B(0 ; H)= & \frac{1}{\Gamma(H+0.5)}\left\{\int _ { - \infty } ^ { 0 } \left[|t-s|^{H-0.5}\right.\right. \\
& \left.-|s|^{H-0.5}\right] \mathrm{dB}(s) \\
& \left.+\int_{0}^{t}|t-s|^{H-0.5} \mathrm{~dB}(s)\right\}
\end{aligned}
$$

where $H$ denotes the fractal scaling parameter (fractal dimension); in this example, we take $H=0.8$, and $\Gamma(\cdot)$ is the Gamma function. The nonstationarity of FBM processes can be represented by the expectation with time difference defined as [17]

$$
E_{s}[B(n+k, H)-B(n, H)]^{2}=k^{2 H} V_{H}
$$

where $V_{H}=\left[\Gamma\left(H+\frac{1}{2}\right)\right]^{-2}\left\{\int_{-\infty}^{0}\left[(1-s)^{H-\frac{1}{2}}-(-s)^{H-\frac{1}{2}}\right]^{2}\right.$ $\left.d s+\frac{1}{2 H}\right\}$. Hence

$$
R_{B, B}(k, n)=\{(n+k) n\}^{H} \frac{0.9585}{\Gamma^{2}\left(H+\frac{1}{2}\right)}
$$

and

$$
\mathrm{AF}_{s, s}(k, n)=\frac{1}{2}\left(1-2^{1-2 H} \cos (2 k n)\right) \frac{1}{\frac{n}{2} 1+2 H} .
$$

Case 1: The additive noise is the zero mean white Gaussian noise. Substituting the above statistic characteristics into the optimal reconstruction filter (46), we obtain Wiener filter $\hat{F}(k, z)$. Then, $\hat{F}(k, z)$ is embedded in the AF filter bank to reconstruct the transmitted FBM signal. The $\overline{\mathrm{SNR}_{r}}$ for different $\mathrm{SNR}_{s}$ 's is obtained from 100 Monte Carlo simulations for 30 realizations of different FBM signals. For example, with $\mathrm{SNR}_{s}=20 \mathrm{~dB}, \overline{\mathrm{SNR}_{r}}=15.5223 \mathrm{~dB}$ is obtained. A typical realization with $\mathrm{SNR}_{r}=18.2946 \mathrm{~dB}$ is illustrated in Fig. 6 . 


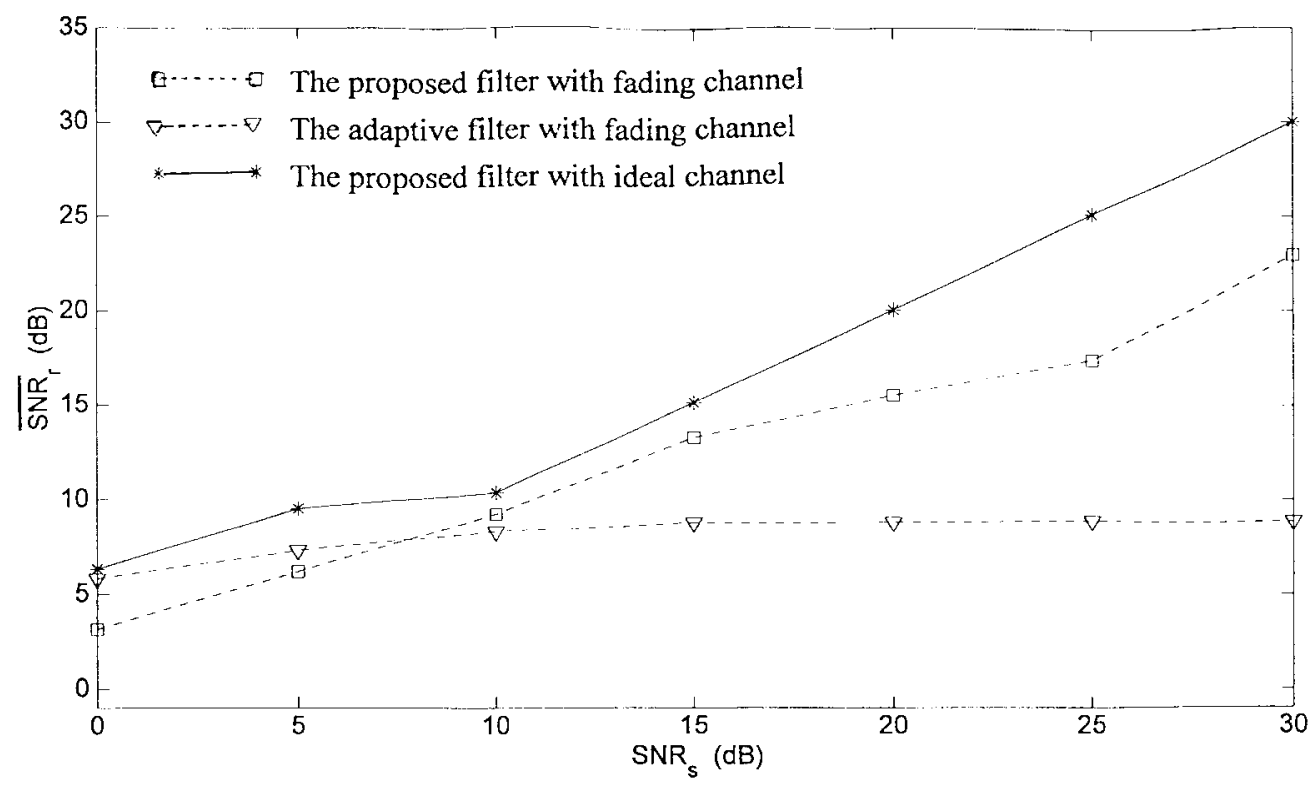

(a)

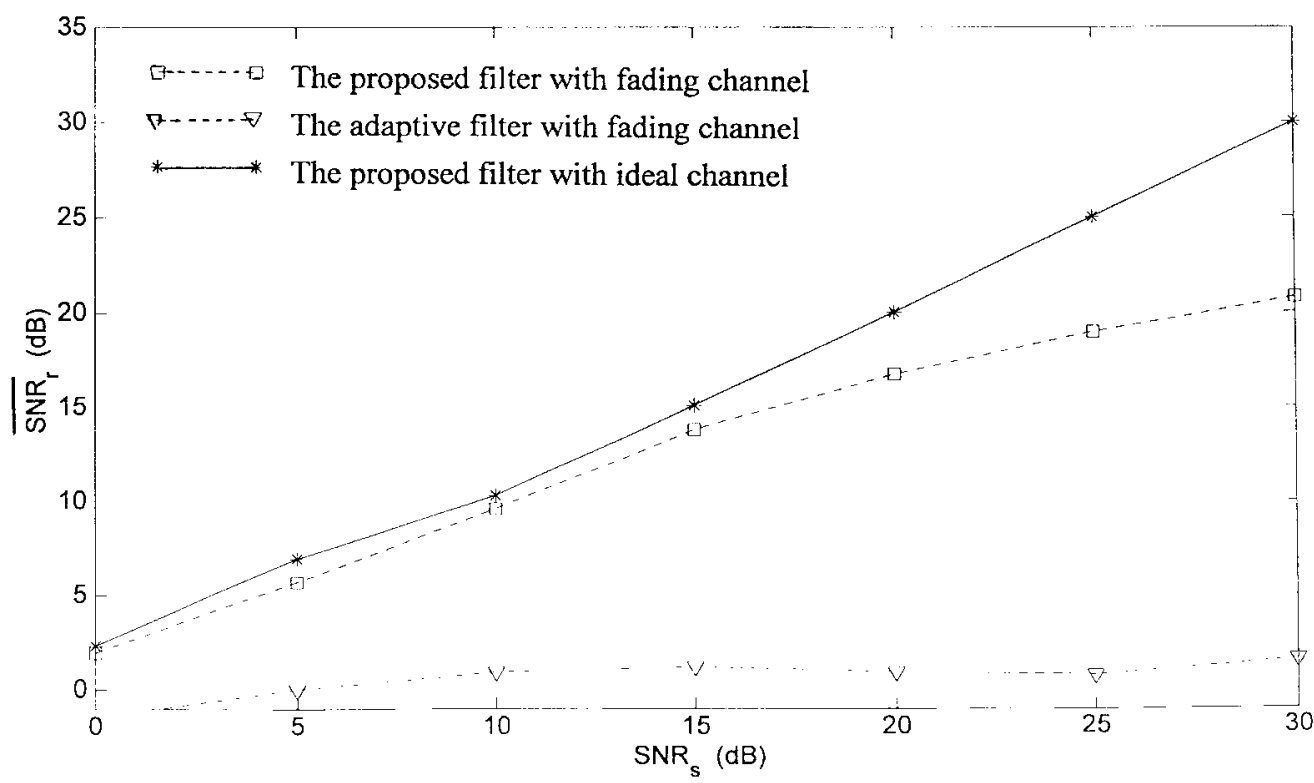

(b)

Fig. 8. (a) Comparison of $\mathrm{AF}$ method with conventional adaptive method in Example 1. (b) Comparison of AF method with conventional adaptive method in Example 1.

Case 2: In the second case, we consider the FBM signal passing through the same fading channel and corrupted by the frequency jammer $\nu(n)=\cos \left(n+\theta_{0}\right)+\sin (2 n)$, where $\theta_{0}$ is the initial phase uniformly distributed over $(0, \pi]$. It is evident that $E(\nu(n))=0$ for all $n$. Similarly, we can easily obtain the optimal reconstruction filter $\hat{F}(k, z)$ via (46), embedded in the AF filter bank to reconstruct the transmitted FBM signal. For example, with $\mathrm{SNR}_{s}=20 \mathrm{~dB}, \overline{\mathrm{SNR}_{r}}=16.9533 \mathrm{~dB}$. A typical reconstructed signal for $\mathrm{SNR}_{s}=20 \mathrm{~dB}$ (in this realization, $\theta_{0}=-0.1913$ ) with $\mathrm{SNR}_{r}=18.7248 \mathrm{~dB}$ is realized in Fig. 7. It is obvious that the reconstructed signal is very close to the transmitted FBM signal.

For comparison, a FIR adaptive filter [28] with ten tap coefficients is used, with a parameter training period of 2000 samples and a delay of 20 samples. A comparison of reconstruction performance between the proposed method and adaptive filter in the first case is shown in Fig. 8(a). In the case of high power noise, the adaptive algorithm has better performance than the proposed method under fading effects. This indicates that the performance of the proposed method is easily deteriorated by high power noise. However, in the case of low power noise, the performance of the proposed method is much better than that of the adaptive algorithm. Obviously, due to the time-varying property of signal and fading channel effect, the tracking ability of the adaptive filter is restricted, and the performance of its signal reconstruction is deteriorated. However, the proposed method is more robust under nonstationary signal and fading channels. In the second 


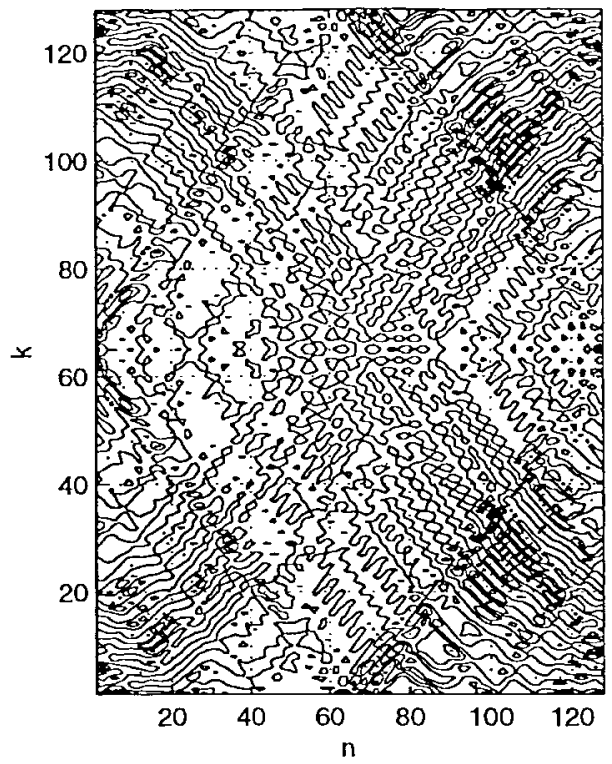

(a)

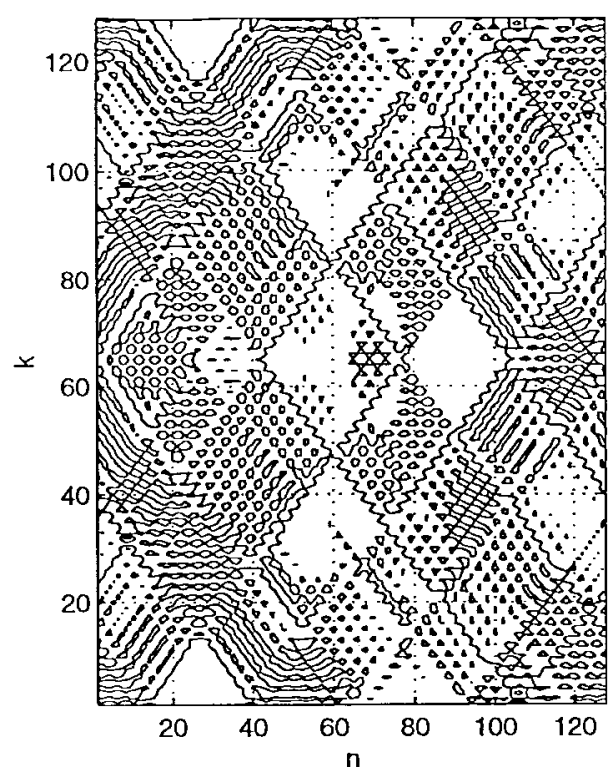

(b)

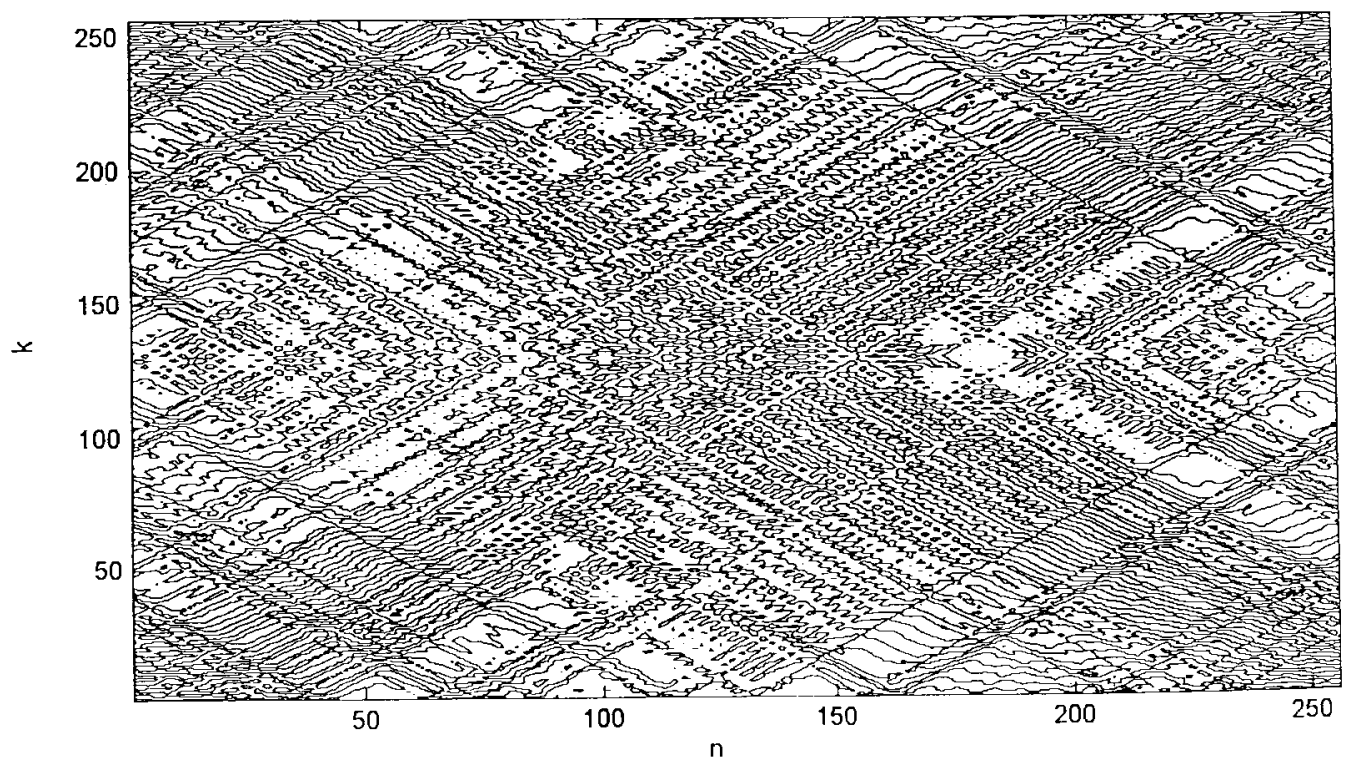

(c)

Fig. 9. Contour plots of $\mathrm{AF}_{s, s}$ and $\mathrm{AF}_{\nu, \nu}$ in Example 2. (a) Contour of $\mathrm{AF}_{s, s}$. (b) Contour of $\mathrm{AF}_{\nu, \nu}$. (c) Contour of $\mathrm{AF}_{s+\nu, s+\nu}$.

case (i.e., jammer case), the comparison of the proposed method with the conventional adaptive algorithm is shown in Fig. 8(b). Admittedly, the proposed method is much better than the conventional adaptive algorithm.

Remark: Note that the reconstruction errors are mainly due to the effects of the time variance of the multipath fading channel, corrupted additive noise, and computation error, etc. With an ideal channel $\left(H\left(q^{-1} ; n\right) \equiv 1\right)$, the reconstruction performance is better than that of the fading case, for example, with $\mathrm{SNR}_{s}=20 \mathrm{~dB}, \overline{\mathrm{SNR}_{r}}=20.0369 \mathrm{~dB}$ and $\overline{\mathrm{SNR}_{r}}=$ $20.0354 \mathrm{~dB}$ in cases 1 and 2, respectively. The simulation results are depicted in Fig. 8(a) in the first case and Fig. 8(b) in the second case, respectively. Moreover, in order to discuss the effect of channel fading on the reconstruction performance, we also present the design performance under different fading parameters $\alpha_{i}(i=1,2)$ with $\mathrm{SNR}_{s}=20 \mathrm{~dB}$ (for the limitation of plotting, $\alpha_{0}$ is assumed to be $1, \alpha_{1}$ ranges from 0.2 to 1 with a step size 0.1 , and $\alpha_{2}$ ranges from 0.03 to 0.4 with a step size 0.01). The results are shown in Fig. 13.

Example 2-Time-Varying Signal Separation Case: Let $\theta_{0}$ and $\theta_{1}$ be independent uniform random variables between 0 and $2 \pi$. We define two stochastic processes [26] as

$$
\begin{aligned}
& s(n)=\sin \left(n^{2} / N+\theta_{0}\right)+\sin \left(n^{3}\right) \\
& \nu(n)=\cos \left(\pi p(n)+\theta_{1}\right)+\sin (n)
\end{aligned}
$$

where $p(n)=\frac{1}{2} m_{f} n^{2}+f_{1} n$ is the phase function. The instantaneous frequency $m_{f} n+f_{1}$ is a linear function of $n$ with slope $m_{f}$ and initial frequency $f_{1}$. We intend to separate $s(n)$ from the multicomponent signal $y(n)=s(n)+\nu(n)$. 


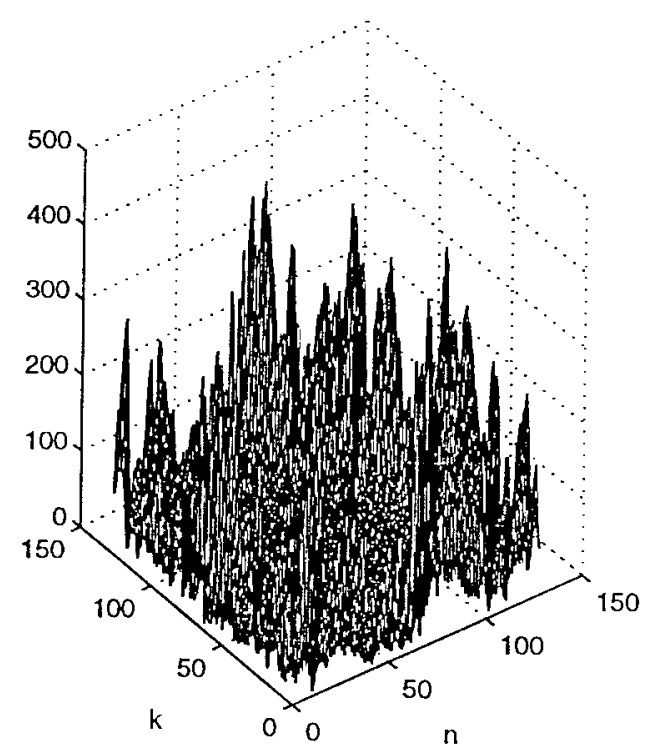

(a)

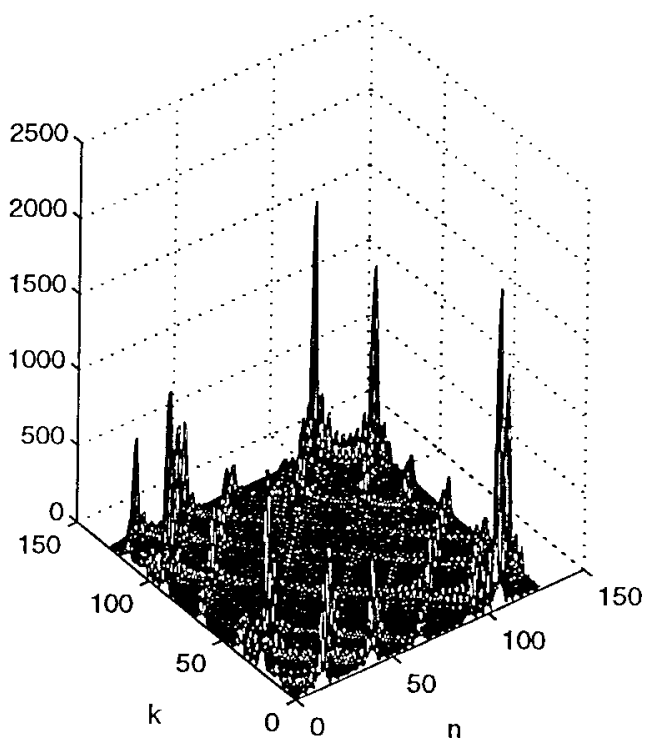

(b)

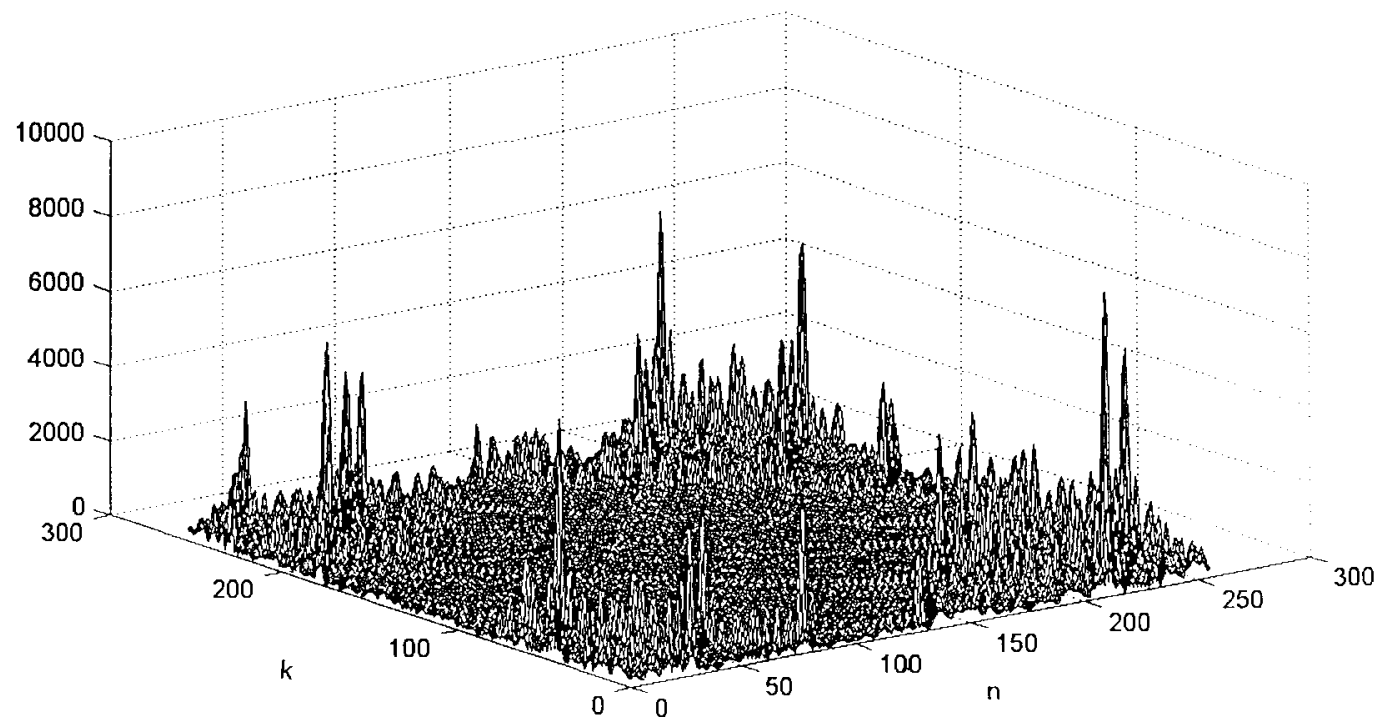

(c)

Fig. 10. Mesh plots of $\mathrm{AF}_{s, s}$ and $\mathrm{AF}_{\nu, \nu}$ in Example 2. (a) Mesh of $\mathrm{AF}_{s, s}$. (b) Mesh of $\mathrm{AF}_{\nu, \nu}$. (c) $\mathrm{Mesh}_{\mathrm{of}} \mathrm{AF}_{s+\nu, s+\nu}$.

Here, $E(\nu(n))=0$ for all $n$

$$
R_{s, s}(k, n)=\frac{1}{2} \cos \left(\frac{k^{2}+2 n k}{N}\right)+\sin \left(n^{3}\right) \sin \left((n+k)^{3}\right)
$$

and

$R_{\nu, \nu}(k, n)=\sin (n) \sin (n+k)-\frac{1}{2} \cos (\pi p(n)-\pi p(n+k))$.

Thus, $\mathrm{AF}_{s, s}(k, n)$ and $\mathrm{AF}_{\nu, \nu}(k, n)$ can be obtained. $N$ is chosen to be $256, m_{f}=0.6$, and $f_{1}=1$. The contour and 3-D mesh plots of $\mathrm{AF}_{s, s}(k, n)$ (in this realization, $\theta_{0}=$ $.2704), \mathrm{AF}_{\nu, \nu}(k, n)$ (in this realization, $\theta_{1}=-0.4671$ ) and $\mathrm{AF}_{y, y}(k, n)$ are given in Figs. 9 and 10, respectively. We see that the domains of their ambiguity distributions are highly overlapped. It is not easy to employ a conventional mask filter in the time-frequency domain to separate $s(n)$ and $\nu(n)$ in this case. The proposed method is one feasible solution for this class of time-varying signal separation problem.

The simulation result reveals that for equal power $\left(\mathrm{SNR}_{s}=\right.$ $0 \mathrm{~dB}), \overline{\mathrm{SNR}_{r}}=2.2832 \mathrm{~dB}$. In the case of $\mathrm{SNR}_{s}=20 \mathrm{~dB}$, $\overline{\mathrm{SNR}_{r}}=20.009 \mathrm{~dB}$. A typical realization for $\mathrm{SNR}_{s}=0$ $\mathrm{dB}$ (in this realization, $\theta_{0}=.4671, \theta_{1}=0.2704$ ) with $\mathrm{SNR}_{r}=2.4064 \mathrm{~dB}$ and $\mathrm{SNR}_{s}=20 \mathrm{~dB}$ (in this realization, $\left.\theta_{0}=.5311, \theta_{1}=-.7545\right)$ with $\mathrm{SNR}_{r}=20.0368 \mathrm{~dB}$ is illustrated in Fig. 11(a)-(d), respectively. This is a rather good reconstruction.

For comparison, a FIR adaptive filter with ten tape coefficients is used. In each realization of $M_{1}$ simulations, a parameter training period of 2000 samples and a delay of 20 samples are used to obtained these tape coefficients, and the estimated parameters are used to simulate $M_{2}$ different sets of noises. A comparison of separation performance between the 


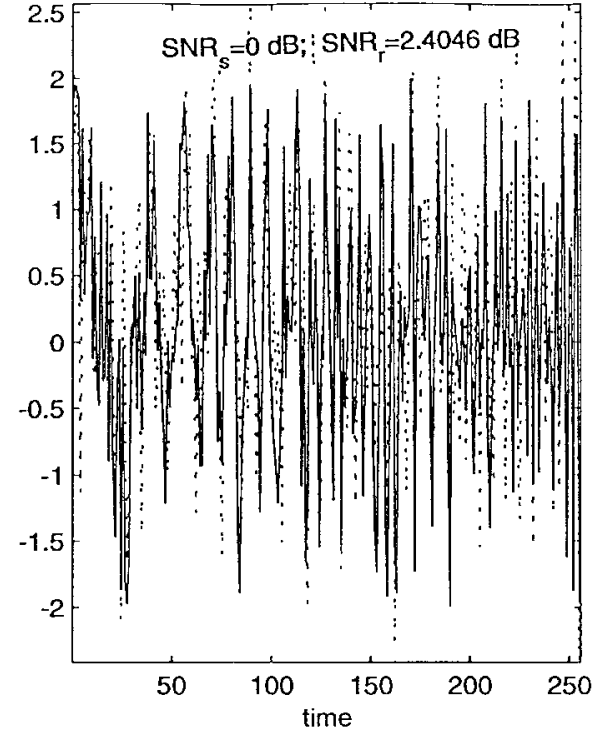

(a)

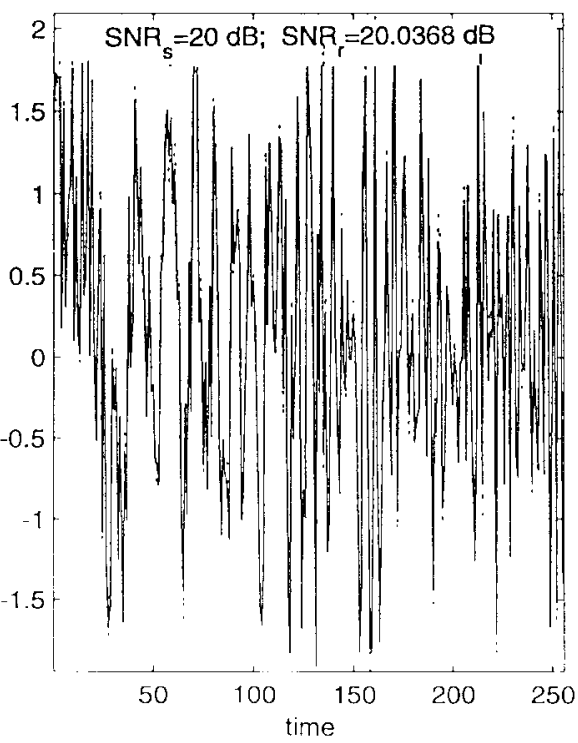

(c)

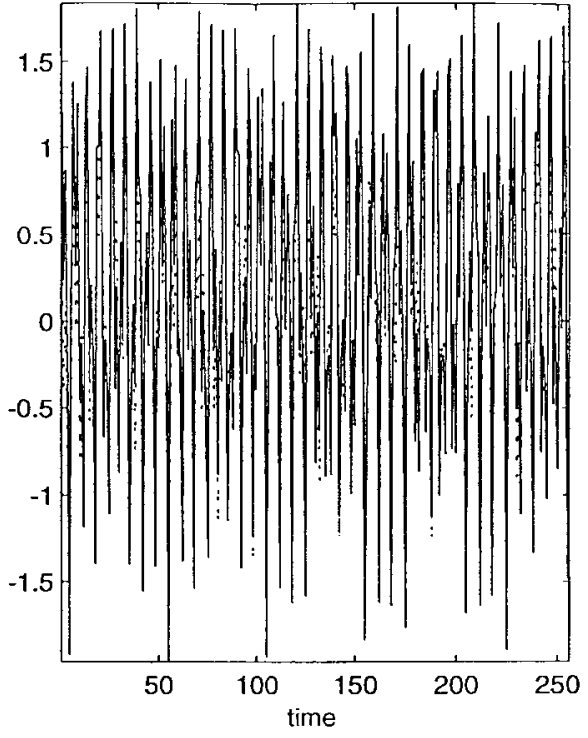

(b)

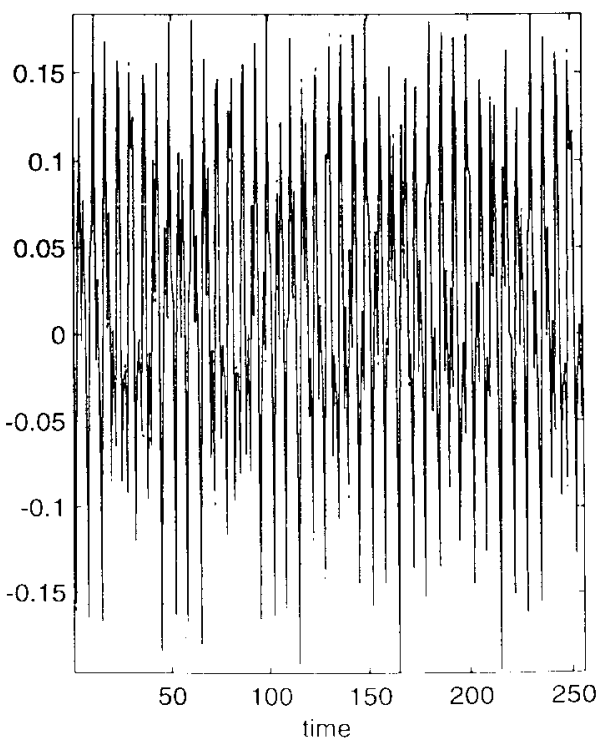

(d)

Fig. 11. Signal separation in Example 2. (a), (b) $\mathrm{SNR}_{s}=0 \mathrm{~dB}, \mathrm{SNR}_{r}=2.4046 \mathrm{~dB}$ (c), (d) $\mathrm{SNR}_{s}=20 \mathrm{~dB}, \mathrm{SNR}_{r}=20.0368 \mathrm{~dB}$. (a) $s(n)$ versus $\hat{s}(n)$. (b) $v(n)$ versus $\hat{v}(n)$. (c) $s(n)$ versus $\hat{s}(n)$. (d) $v(n)$ versus $\hat{v}(n)$.

proposed method and adaptive filter is shown in Fig. 12. Since the nonstationary property of signal and noise is removed by the AF filter bank and optimal reconstruction is considered in the design procedure, the performance of the proposed method is better than that of the adaptive filter. Obviously, the tracking ability of adaptive algorithm is deteriorated by the fast time-varying signals.

\section{CONCLUSION}

An AF filter bank has been developed for the first time to treat the optimal deconvolution problem of nonstationary signal transmission through a multipath fading channel under nonstationary noise. Unlike conventional studies of signal analysis/synthesis via AF [5], this study focuses on the analysis/synthesis of a nonstationary signal transmission system with corrupted noise. Optimization techniques such as calculus of variation, spectral factorization, etc., are employed to obtain a stable and causal Wiener filter bank to achieve optimal estimation of the transmitted signal. The optimal separation problem for multicomponent nonstationary signals is a special case of our design. Based on AF analysis/synthesis techniques, a new time-frequency deconvolution filter design is proposed using an AF filter bank equipped with a Wiener filter bank for signal reconstruction in a nonstationary (or time-varying) signal transmission system through a multipath fading channel.

Furthermore, in the design procedure of optimal Wiener filter bank, the statistical properties of the multipath fading channel have been also considered in the MSE sense. Therefore, the proposed time-scale deconvolution filter bank can achieve the optimal signal reconstruction under the environment of channel fadings. 


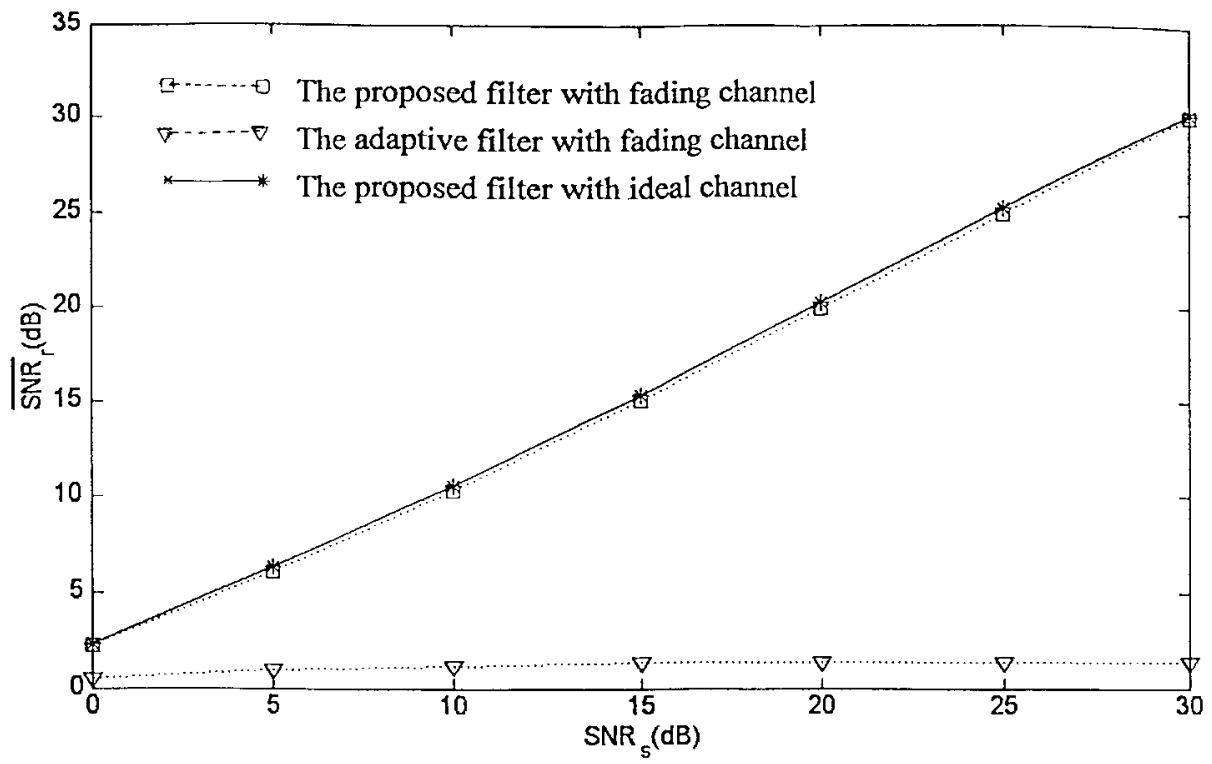

Fig. 12. Comparison of AF method with conventional adaptive method in Example 2.

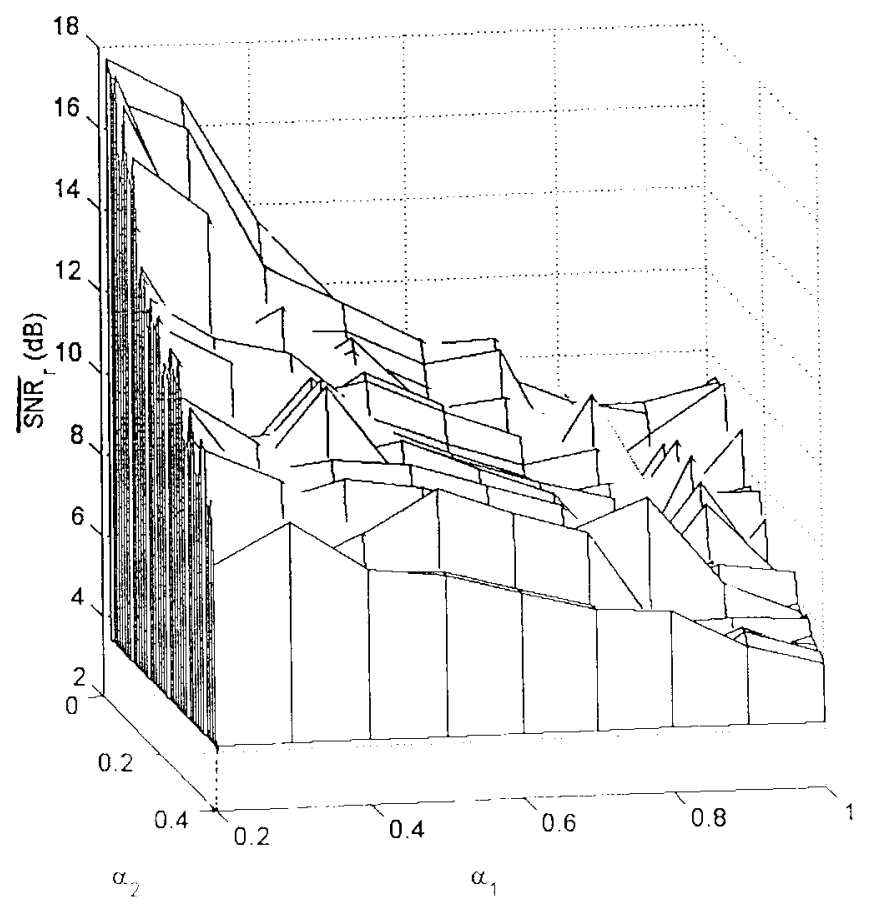

Fig. 13. Performance with different $\alpha_{1}$ and $\alpha_{2}$ in Example $1\left(\mathrm{SNR}_{s}=20\right.$ $\mathrm{dB})$.

Unlike the adaptive design method, which must be updated in every training cycle, the proposed time-frequency deconvolution filter is developed with a closed form for nonstationary signal transmission through a multipath fading channel. Furthermore, the channel noise is not restricted to be white Gaussian; it may be nonstationary or time varying. Therefore, the proposed AF filter bank-based deconvolution filter may find more applications in the fields of radar and sonar transmission systems, acoustic and speech transmission systems, and communication systems. From the simulation results, we have found that the proposed time-frequency deconvolution filter design has very good performance.

\section{ACKNOWLEDGMENT}

The authors would like to thank the reviewers and associate editor for their constructive comments and suggestions, which have greatly improved the quality of this manuscript.

\section{REFERENCES}

[1] L. Auslander and R. Tolimieri, "Computing decimated finite crossambiguity functions," IEEE Trans. Acoust., Speech, Signal Processing, vol. 36, pp. 359-363, Mar. 1988

[2] S. Nenedetto, E. Biglieri, and V. Castellani, Digital Transmission Theory. Englewood Cliffs, NJ: Prentice-Hall, 1989.

[3] W. Blau, "Synthesis of ambiguity functions for prescribed responses," IEEE Trans. Aerosp. Electron. Syst., vol. AES-3, pp. 656-663, July 1967.

[4] M. G. Amin, "Time-frequency spectrum analysis and estimation for nonstationary random process," in Time-Frequency Signal Analysis, B. Boualem, Ed. New York: Wiley, 1992, pp. 208-232, ch. 9.

[5] G. F. Boudreaux-Bartels and T. W. Parks, "Time-varying filtering and signal estimation using Wigner distribution synthesis techniques," IEEE Trans. Acoust., Speech, Signal Processing, vol. ASSP-34, pp. 442-451, June 1986

[6] R. D. Buda, "Signals that can be calculated from their ambiguity function," IEEE Trans. Inform. Theory, vol. IT-16, pp. 195-202, Mar. 1970.

[7] B. S. Chen and S. C. Peng, "Optimal deconvolution filter design based on orthogonal principle," Signal Process., vol. 25, pp. 361-372, 1991.

[8] B. S. Chen and C. W. Lin, "Multiscale Wiener filter for the restoration of the fractal signal: Wavelet filter bank approach," IEEE Trans. Signal Processing, vol. 42, pp. 2972-2982, Nov. 1994.

[9] Y. L. Chen and B. S. Chen, "Minimax robust deconvolution filter under stochastic parametric and noise uncertainties" IEEE Trans. Signal Processing, vol. 42, pp. 32-45, Jan. 1994.

[10] L. Cohen, "Time-frequency distribution-A review," Proc. IEEE, vol. 77, pp. 941-981, July 1989.

[11] L. Cohen, Time-Frequency Analysis. Englewood Cliffs, NJ: PrenticeHall, 1995.

[12] C. E. Cook and M. Bernfeld, Radar Signals: An Introduction to Theory and Application. Boston, MA: Artech House, 1993.

[13] P. Flandrin, "On the spectrum of fractional Brownian motions," IEEE Trans. Inform. Theory, vol. 35, pp. 197-199, Jan. 1989.

[14] F. Hlawatsch and G. F. Boudreaux-Bartels, "Linear and quadratic timefrequency signal representation," IEEE Signal Processing Mag., vol. 9, pp. 21-67, Apr. 1992.

[15] V. Kucera, Discrete Linear Control: The Polynomial Equation Approach. New York: Wiley, 1979.

[16] T. Lundahl, W. J. Ohley, S. M. Key, and R. Siffert, "Fractional Brownain motion: A maximum likelihood estimator and its application to image texture," IEEE Trans. Med. Imag., vol. MI-5, pp. 152-161, Sept. 1986. 
[17] B. B. Mandelbrot and J. W. Van Ness, "Fractional Brownian motions, fractional noise and application," SIAM Rev., vol. 10, no. 4, Oct. 1968.

[18] J. M. Mendel, Optimal Seismic Deconvolution: An Estiomation-Based Approach. New York: Academic, 1983.

[19] T. J. Moir, "Optimal deconvolution smoother," Proc. Inst. Elect. Eng., vol. 133, pt. D, no. 1, pp. 13-18, Jan. 1986.

[20] A. V. Oppenheim and R. W. Schafer, Discrete-Time Signal Processing. Englewood Cliffs, NJ: Prentice-Hall, 1989.

[21] J. G. Proakis, Digital Communications, 3rd ed. New York: McGrawHill, 1995.

[22] A. W. Rihaczek, "Signal energy distribution in time and frequency," IEEE Trans. Inform. Theory, vol. IT-14, pp. 269-274, May 1968.

[23] S. M. Sussman, "Least-square synthesis of radar ambiguity functions," IRE Trans. Inform. Theory, vol. IT-9, pp. 246-254, Apr. 1962.

[24] B. V. K. V. Kumar, C. P. Neuman, and K. J. Devos, "Discrete wigner synthesis," Signal Process., vol. 11, pp. 277-304, 1986.

[25] K. B. Yu and S. Cheng, "Signal synthesis from wigner distribution," in Proc. IEEE Int. Conf. Acoust., Signal Process., Tampa, FL, Mar. 1985, pp. 1037-1040.

[26] F. Deprettere, Ed., SVD and Signal Processing. Amsterdam, The Netherlands: North-Holland, 1988.

[27] R. B. Ash, Topics in Stochastic Process. New York: Academic, 1975.

[28] V. K. Ingle and J. G. Proakis, Digital Signal Processing. New York: PWS, 1997

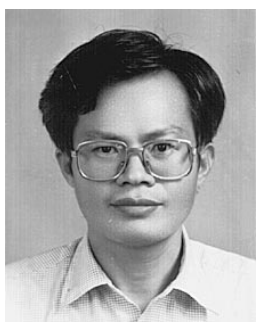

Bor-Sen Chen (M'82-SM'89) received the B.S degree from Tatung Institute of Technology, Taiwan, R.O.C., in 1970, the M.S. degree from National Central University, Taiwan, in 1973, and the Ph.D. degree from the University of Southern California, Los Angeles, in 1982.

He was a Lecturer, Associate Professor, and Professor at Tatung Institute of Technology from 1973 to 1987 . He is now a Professor at National Tsing Hua University, Hsin-Chu, Taiwan. His current research interests include control and signal process-

ing.

Dr. Chen has received the Distinguished Research Award from the National Science Council of Taiwan four times.

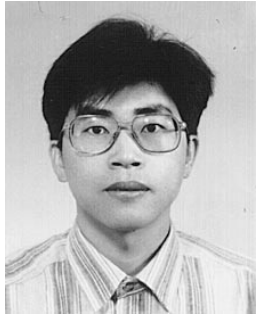

Yue-Chiech Chung was born on July 31, 1971 in Miali, Taiwan, R.O.C. He received the B.S. degree from the Department of Control Engineering, National Chiao-Tung University, Taiwan, in 1994 and the M.S. degree from the Department of Electrical Engineering, National Tsing-Hua University, HsinChu, Taiwan, in 1996.

His research interests are in digital signal process ing algorithms and the applications of the ambiguity function.

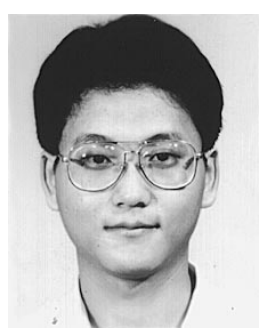

Der-Feng Huang received the B.S. degree from Tunghai University, Taichung, Taiwan, R.O.C., and the M.S. degree from Cheng Kung University, Tainan, Taiwan, both in mathematics, in 1990 and 1992, respectively. Presently, he is working toward the Ph.D. degree in electrical engineering at National Tsing Hua University, Hsin-Chu, Taiwan.

His current research interests include digital signal processing, time-frequency representation, and time-varying filtering. He is also teaching at the National Yang Ming University. 